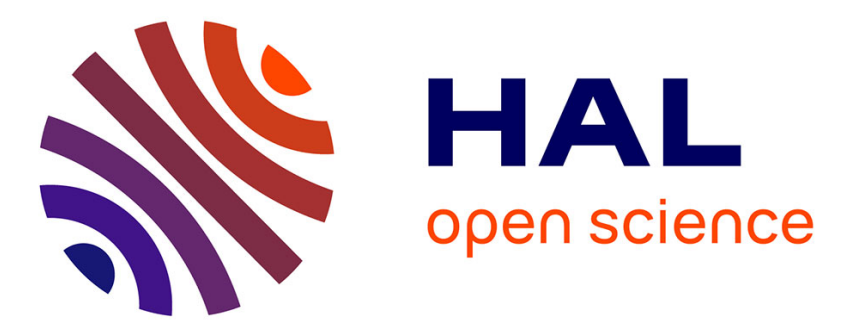

\title{
Electron beam charging of insulators: A self-consistent flight-drift model
}

Matthieu Touzin, Dominique Goeuriot, Christelle Guerret-Piécourt, Denyse Juvé, Daniel Tréheux, Hans-Joachim Fitting

\section{- To cite this version:}

Matthieu Touzin, Dominique Goeuriot, Christelle Guerret-Piécourt, Denyse Juvé, Daniel Tréheux, et al. Electron beam charging of insulators: A self-consistent flight-drift model. Journal of Applied Physics, 2006, 99 (11), pp.114110. 10.1063/1.2201851 . hal-00163640

\section{HAL Id: hal-00163640 https://hal.science/hal-00163640}

Submitted on 23 Jul 2007

HAL is a multi-disciplinary open access archive for the deposit and dissemination of scientific research documents, whether they are published or not. The documents may come from teaching and research institutions in France or abroad, or from public or private research centers.
L'archive ouverte pluridisciplinaire HAL, est destinée au dépôt et à la diffusion de documents scientifiques de niveau recherche, publiés ou non, émanant des établissements d'enseignement et de recherche français ou étrangers, des laboratoires publics ou privés. 


\section{Electron Beam Charging of Insulators - \\ a Selfconsistent Flight-Drift Model}

M. Touzin and D. Goeuriot

Centre Sciences des Matériaux et des Structures, Ecole Nationale Supérieure des Mines, 158 cours Fauriel, F - 42023 Saint-Etienne cedex 2, France

C. Guerret-Piécourt, D. Juvé and D. Tréheux

Laboratoire d'Ingénierie et Fonctionnalisation des Surfaces, UMR-CNRS 5621,

Ecole Centrale de Lyon, 36 avenue Guy de Collongue, F - 69134 Ecully cedex, France

H.-J. Fitting*

Physics Department, University of Rostock, Universitätsplatz 3, D - 18051 Rostock, Germany

(Received)

* Corresponding author:

Hans-Joachim Fitting

Physics Department, University of Rostock

D - 188051 Rostock

Germany

EMail: hans-joachim.fitting@uni-rostock.de

Tel: (+49)-381-498 6760, Fax: (+49)-381-498 6802 


\section{ABstract}

Electron beam irradiation and the selfconsistent charge transport in bulk insulating samples are described by means of a new flight-drift model (FDM) and an iterative computer simulation. Ballistic secondary electron and hole transport is followed by electron and hole drift, their possible recombination and/or trapping in shallow and deep traps. The trap capture cross sections are Poole-Frenkel-like temperature- and field-dependent. As a main result the spatial distributions of currents $j(x, t)$, charges $\rho(x, t)$, the field $F(x, t)$ and the potential slope $V(x, t)$ are obtained in a selfconsistent procedure as well as the time dependent secondary electron emission rate $\sigma(t)$ and the surface potential $V_{0}(t)$. For bulk insulating samples the time-dependent distributions approach the final stationary state with $j(x, t)=$ const $=0$ and $\sigma=1$. Especially for low electron beam energies $E_{0}<4 \mathrm{keV}$ the incorporation of mainly positive charges can be controlled by the potential $V_{G}$ of a vacuum grid in front of the target surface. For high beam energies $E_{0}=10 \mathrm{keV}, 20 \mathrm{keV}$, and $30 \mathrm{keV}$ high negative surface potentials $V_{0}=-4 \mathrm{kV},-14 \mathrm{kV}$, and $-24 \mathrm{kV}$ are obtained, respectively. Besides open non-conductive samples also positive ion-covered samples and targets with a conducting and grounded layer (metal or carbon) on the surface have been considered as used in environmental scanning electron microscopy (ESEM) and common SEM in order to prevent charging. Indeed, the potential distributions $V(x)$ are considerably small in magnitude and do not affect the incident electron beam neither by retarding field effects in front of the surface nor within the bulk insulating sample. Thus the spatial scattering and excitation distributions are almost not affected. 


\section{INTRODUCTION}

Insulating and dielectric materials, especially as oxides, perovskites, ceramics, and functional layers become more and more important in modern technology, ${ }^{1}$. Especially, the influence of dielectric polarization and charging on the features of these materials has been investigated more intensively and reported, e.g. on the conference series on Electric Charges in Non-Conductive Materials ${ }^{2,3}$. Furthermore, the electrical charging of insulators under different types of ionizing irradiation (electrons, neutrons, and X- $\gamma$-Rays) is of considerable interest in many fields of technology and science from the development of thermonuclear fusion (ITER) as a possible future source of energy, see Ref. 4, to the multiform development of insulating materials for satellites and spacecrafts protection ${ }^{5}$. All these applications come within the same physical mechanism. Irradiation induces the injection of high energetic charges and generates electron-holes pairs. Secondary electrons are emitted but an important part of the charge carriers remains in the sample and its drift depends on the trapping properties of the material. The knowledge of these charging phenomena would help in preventing insulator breakdown mainly responsible for the damage of electronic devices $^{6}$. Moreover, the electrical charging phenomena also play a major role in modern analytical techniques like Scanning Electron Microscopy (SEM),Environmental one's (ESEM), Auger Electron Spectroscopy (AES), electron energy loss spectroscopy (EELS) etc., when investigating non-conductive samples. Indeed, the prediction of the influence of charging is essential to interprete the results of analysis ${ }^{7-9}$. The strong charging of insulators under electron beam irradiation is well known, at least, since Malter 1936 discovered the anomalous high secondary electron emission (SEE) and long-lasting electron post-emission from $\mathrm{MgO}$ layers ${ }^{10}$. A strong positive charging due to the emission of secondary electrons (SE) from the 
surface-near regions is responsible for that selfconsistent field-enhanced SEE. On the other hand, the deeper injection of primary electrons (PE) will produce an electron surplus within the bulk of an insulator. The respective charge $\rho(x)$ and field $\boldsymbol{F}(x)$ distributions maintain the selfconsistent charge transport and the SEE emission.

An approach for a first estimation is based on the dynamic double layer model (DDLM) in which the phenomenon is brought to the simplified case of two layers of opposite charge. Complete solutions of the respective equations were achieved by Melchinger et al. ${ }^{11}$. More recently, J. Cazaux ${ }^{12}$ developed an effective approach of the SEE evolution in insulating samples using this DDLM.

The first comprehensive Monte Carlo calculations of the self-consistent charging were made by Vicario et al. ${ }^{13}$, Ganachaud et al. ${ }^{14}$ and Renoud et al. ${ }^{15,16}$. Of course, these calculations are complex because of the deal with the full simulation of primary electron straggling as well as with the generation and transport of secondary electrons and holes in the selfconsistent field. However, the decisive advantage of the full Monte-Carlo simulation is the 3-dimensional description of the charging process with the lateral charge spreading in case of point-like electron beam injection by a very small beam focus. Thus, the above mentioned authors $^{13,15}$ could demonstrate the charge build-up by a computer animation.

Nevertheless, it is of importance to precise the types of theory that have been led to enlighten this phenomenon of selfconsistent charging. One of the first attempts was the planar selfconsistent charging simulation of our co-author (HJF) already in $1978^{17,18}$, later on improved for insulating layers on conducting substrate in Ref. 19 and for bulk materials in Ref. 10. These authors use field-dependent attenuation lengths $\lambda(F)$ for the ballistic transport of electrons and holes which had been found experimentally by means of electron beam induced currents (EBIC) measurements ${ }^{21,22}$ and had been verified by Monte Carlo 
calculations $^{23-26}$.

Of course, the planar 1-dimensional self-consistent model will approach validity if the electron beam diameter is much larger than the maximum electron penetration depth $R\left(E_{0}\right)$. Unambiguously, this is fulfilled in scanning electron microscopes (SEM) with a slightly defocused beam. Thus the 1-dimensional simulation can be applied to 3-dimensional description of the sample potential in a SEM chamber ${ }^{27}$.

The present paper will extend the ballistic flight model for electrons and holes to a more comprehensive and realistic new flight-drift model (FDM). There the ballistic flight of excited electrons and holes is followed by their drift and respective recombination and/or trapping in shallow and deep traps. Three types of non-conductive samples have been taken into account: A - the insulating bulk sample with an open surface to the vacuum, B - the bulk sample with a positive ion-covered surface as it is given in environmental secondary electron microscopy (ESEM) under a certain gas pressure (some Torr) and gas ionization ${ }^{28,29}$, and C - the conducting and grounded surface coated by a metal or carbon layer in order to prevent charging of the bulk insulator. Nevertheless, in the two latter cases strong internal electric fields will appear within the bulk insulator beneath the surface.

The numerical and experimental results will be presented in particular for bulk alumina samples but could be easily adapted to any insulator using the relevant material data. 


\section{THEORETICAL BACKGROUND}

The problem of electron beam charge injection in different target arrangements is demonstrated in Fig. 1. Incident electrons (so-called primary electrons PE) with initial energy $E_{0}$ and current density $j_{0}$ penetrate the insulator target up to the maximum range $R\left(E_{0}\right)$ and create a mainly positive-negative spatial charge distribution $\varrho(x)$ where the positive charge beneath the surface is due to secondary electron (SE) escape into vacuum (sample A) or into an electrode upon the surface (sample C). Moreover, a surface coverage by positive ions (sample B) becomes also possible when irradiating the insulating sample in residual gas pressure as we have already mentioned in context with environmental scanning electron microscopy (ESEM) techniques ${ }^{28,29}$ in the introduction. According to the electrode arrangements $\mathrm{A}, \mathrm{B}$, or $\mathrm{C}$ the charges will create different field and potential distributions $F(x)$ and $V(x)$, respectively, with a low surface field $F(x=0) \simeq 0$ and a high floating surface potential $V_{0}$ for the sample (A); and a low surface potential $V_{0}$ for ion-covered surfaces of sample (B) and the grounded surface $V_{0}=0$ of sample $(\mathrm{C})$, but both the latter ones show high electric fields beneath the surface, see Fig. 1 : samples A, B, C.

The generated secondary electrons $(\mathrm{SE})$ and holes $(\mathrm{H})$ will be redistributed by the respective fields $F(x)$ and potentials $V(x)$ maintaining the selfconsistent charge transport in a planar (1-dimensional) model:

$$
\begin{gathered}
\operatorname{div} \mathbf{j}(x, t)=-\frac{\partial}{\partial t} \rho(x, t) \\
F(x, t)=\frac{1}{\varepsilon_{0} \varepsilon_{r}} \int \rho(x, t) d x \\
V(x, t)=\int F(x, t) d x
\end{gathered}
$$

where the electric field $F$, especially, has a strong feed-back to the current distributions. 
The electric field $F(x, t)$ of Eq. (2) in open samples (A) is calculated by successive summation (integration) of charges beginning from the surface into the bulk, i.e. towards the grounded support, see Fig. 1, because the sample support is the nearest electrode to the incorporated charges, much closer than any other metallic part of a scanning electron microscope (SEM). Thus the electric field of all charges possesses a component into the sample and the field at the surface $F(x=0)$ is nearly zero.

The ion-covered samples (B) are fixed to a low potential $V_{0}$ kept by a permanent ion support. Thus, after each iterative integration of charges, beginning at $x=0$ as in open samples, we have to add the field value $F_{d}=-\left(V_{0, \text { open }}-V_{0, \text { ion }}\right) / d$ to all $F(x)$, where $V_{0, \text { open }}$ is the surface potential calculated first for the open sample, then substracted by the fixed value $V_{0 \text {,ion }}$ of the ion-covered sample and devided by the sample thickness $d$. This procedure has to be done after each cycle $j(x), \rho(x), F(x), V(x)$. Thus it guarantees a constant surface potential $V_{0}=V_{0, \text { ion }}$ with the respective time-dependent spatial distributions: $j(x, t), \rho(x, t), F(x, t)$, and $V(x, t)$.

Of course, the metal-coated sample (C) presents a special case of the previous procedure with the fixed value $V_{o \text {,metal }}=0$ and all field contributions of incorporated charges are directed to the close metallic surface electrode, vice versa, as for open samples where the field components are directed to the support as the closest electrode. But here, in metal-coated samples, the electron penetration depth $R$ of nearly $3 \mu m$ (see Fig. 2) can be neglected with respect to the bulk sample thickness $d$ of millimeters.

Then, let us refer first to the current distributions $j(x, t)$. 


\section{A. Primary electron (PE) scattering}

The injection of primary electrons (PE) and their creation of secondary electrons (SE) and holes $(\mathrm{H})$ are very similar for alumina $\mathrm{Al}_{2} \mathrm{O}_{3}$ and silica $\mathrm{SiO}_{2}$ as we have described already in Refs. 18 - 20 based on empirical results of the electron penetration into and through thin films, see "film-bulk method" in Ref. 30. By means of this method the resulting PE current in dependence on the target depth $x$ and the PE initial energie $E_{0}$ was found:

$$
j_{\mathrm{PE}}\left(x, E_{0}\right)=j_{0}(1-\eta) \exp \left[-4.605\left(\frac{x}{R\left(E_{0}, z\right)}\right)^{p(z)}\right],
$$

with $j_{0}$ as impinging PE current density and the material parameters for $\mathrm{Al}_{2} \mathrm{O}_{3}: \eta \approx 0.2$ the backscattering coefficient, $p=2$ the transmission exponent. A new equation for the maximum range $R\left(E_{0}\right)$ of electrons reached by $1 \%$ of $\mathrm{PE}$ in dependence on their initial energy $E_{0}$ was deduced from experimental data, ${ }^{31-36}$, see Fig. 2. Especially, the direct electron transmission measurements through thin selfsupporting films of $\mathrm{Al}_{2} \mathrm{O}_{3}$ and $\mathrm{SiO}_{2}$ of Vyatskin and Trunev ${ }^{32}$ led to the best fit for $\mathrm{Al}_{2} \mathrm{O}_{3}$ :

$$
R / \mathrm{nm}=28.7\left(E_{0} / \mathrm{keV}\right)^{1.55}
$$

$\mathrm{R}$ is given in $\mathrm{nm}$, and the electron beam energy $E_{0}$ should be inserted in $\mathrm{keV}$. Electron penetration curves according to Eq. (4) and (5) are presented in the upper part of Fig. 3. For an exact charge balance within the insulating target the deposited, i.e. absorbed PE's in the depth $x$ have to be taken into account too. They present an additional electron charge, added to the excited secondary electrons (SE), which are generated in parity to the positive holes $(\mathrm{H})$. The PE absorption density, or better said PE deposition function $g_{\mathrm{PE}}$, is given by the negative first derivative of the PE penetration current of Eq (4):

$$
j_{0} \cdot g_{P E}=-\frac{d j_{P E}}{d x}=j_{0}\left(1-\eta_{R}\right) \frac{4.605}{R^{2}} \cdot 2 x \exp \left[-4.605\left(\frac{x}{R}\right)^{2}\right]
$$


or including the expression for $j_{P E}$ from Eq (4) we get:

$$
j_{0} \cdot g_{P E}=9.21 \cdot \frac{x}{R^{2}} \cdot j_{P E}\left(x, E_{0}\right):
$$

PE deposition functions $g_{P E}\left(x, E_{0}\right)$ are presented in the middle part of Fig. 3. Of course, these exhausted and deposited PE will continue their motion like created common SE and should be added finally to the SE generation function $g_{\mathrm{SE}}$ of Eq. (7) as will be done in Eq. (12).

On the other hand, the spatial SE generation rate $g_{S E}\left(x, E_{0}\right)$ excited by $\mathrm{PE}$ is proportional to the spatial energy loss $d E / d x$ of the impinging and straggling primary electrons (PE), i.e. proportional to the spatial PE energy transfer to the target volume:

$$
g_{S E}\left(x, E_{0}\right)=\alpha \frac{1}{E_{i}} \frac{d E}{d x},
$$

where $E_{i}$ is the mean creation energy for one SE and $\alpha$ a yield factor of nearly a unit.

According to Klein ${ }^{37}$ and Alig and Bloom ${ }^{38}$ the SE creation energy increases with the energy gap $E_{g}$ of a given target material

$$
E_{i} \approx 3 E_{g}+1 \mathrm{eV}
$$

resulting in $E_{i} \approx 28 \mathrm{eV}$ for $\mathrm{Al}_{2} \mathrm{O}_{3}$ with $E_{g}=9 \mathrm{eV}$. Then with Eq. (7) and an empirical expressions for $d E / d x$ from Ref. 30, we may write the $\mathrm{SE}$ generation rate $g_{\mathrm{SE}}$ in $\mathrm{SiO}_{2}$ and $\mathrm{Al}_{2} \mathrm{O}_{3}$ targets in the form of a semi-empirical equation

$$
g_{S E}\left(x, E_{0}\right)=g_{\mathrm{SH}}\left(x, E_{0}\right)=\frac{1.544}{R\left(E_{0}\right)} \frac{E_{0}}{E_{i}} \exp \left[-7.5\left(\frac{x}{R}-0.3\right)^{2}\right]
$$

Of course, secondary electrons $\left(g_{S E}\right)$ and holes $\left(g_{S H}\right)$ are created in parity presenting a Gaussian distribution with the maximum shifted by $0.3 R$ from the surface into the target volume as shown in the bottom part of Fig. 3 .

In general, the electron beam scattering in solid targets has been recently described by six semi-empirical laws ${ }^{39}$ including the Eqs.(4) - (9). 


\section{B. Ballistic currents of secondary electrons and holes}

After generation secondary electrons and holes are flying and straggling ballistically over certain distances, so-called attenuation lengths $\lambda$ depending on the presence of an electric field $F$ and its direction. We have demonstrated this process by Monte Carlo simulation in $^{23-25}$ and measured $\lambda(F)$ directly by electron beam induced currents EBIC, ${ }^{21,22}$. As a main result we get attenuation probabilities $W_{E F}$ for ballistic electrons (E) expressed by mean field-dependent attenuation lengths:

$$
\lambda_{E}(F)=\lambda_{E, 0} \exp \left(-\beta_{E} F\right)
$$

and

$$
W_{E F}=\exp \left(-\frac{\Delta x}{\lambda_{E, 0} \exp \left(-\beta_{E} F\right)}\right)
$$

in $(F>0)$ and against $(F<0)$ field direction. Here $\lambda_{E, 0}$ presents the field-free attenuation length and $-\beta_{E}$ the electron field attenuation parameter. Similar expressions can be written for ballistic holes with $\lambda_{H, 0}$ and $+\beta_{H}$, respectively. In Fig. 4 the field-dependent attenuation lengths $\lambda(F)$ are presented for electrons (E) and holes $(\mathrm{H})$.

Assuming an isotropic SE generation, one half of the created SE: $1 / 2 j_{0} g_{S E}\left(x, E_{0}\right) \Delta x$ will move into the bulk sample, i.e. in the direction towards the sample support, called transmission $(\mathrm{T})$, and the other half towards the sample surface, called reverse direction or remission $(\mathrm{R})$. Then the respective continuity equation in 1-dimensional form for ballistic SE or hole currents in transmission (T) direction towards the sample substrate (holder) or in reverse or remission $(\mathrm{R})$ direction towards the surface can be written ${ }^{19,20}$ :

$$
j_{\mathrm{BET}}^{\mathrm{BER}}(x)=\left[j_{\mathrm{BET}}^{\mathrm{BER}}(x \pm \Delta x)+\frac{1}{2} j_{0}\left[g_{\mathrm{SE}}\left(x, E_{0}^{\prime}\right)+g_{\mathrm{PE}}\left(x, E_{0}^{\prime}\right)\right] \Delta x\right] \underbrace{\exp \left[-\frac{\Delta x}{\lambda_{\mathrm{E}, 0} \exp \left( \pm \beta_{\mathrm{E}} F\right)}\right]}_{W_{\mathrm{EF}}}
$$


and for holes, respectively:

$$
\begin{array}{r}
j_{\mathrm{BHT}}^{\mathrm{BHR}}(x)=\left[j_{\mathrm{BHT}}^{\mathrm{BHR}}(x \pm \Delta x)+\frac{1}{2} j_{0} g_{\mathrm{SE}}\left(x, E_{0}^{\prime}\right) \cdot \Delta x\right] \underbrace{\exp \left[-\frac{\Delta x}{\lambda_{\mathrm{H}, 0} \exp \left(\mp \beta_{\mathrm{H}} F\right)}\right]}_{\text {generation }} \\
\text { convection }
\end{array}
$$

The first term in the brackets presents the convection part from the neighboring cell $\Delta x$; the second one the generation of inner SE: $\left(g_{\mathrm{SE}}+g_{\mathrm{PE}}\right)$ or holes $\left(g_{\mathrm{SH}}=g_{\mathrm{SE}}\right)$ followed by the ballistic attenuation probability $W_{\mathrm{EF}}(x)$ and $W_{\mathrm{HF}}(x)$ of the charge carriers over the small distance $\Delta x$ in the target depth $x$. These attenuation probabilities have been described by Eqs. (10) and (11). Additional to Refs. 19,20, here in Eq. (12) we have added the deposited PE with $g_{\mathrm{PE}}$ to the generated SE: $g_{\mathrm{SE}}$.

The part of ballistic SE moving towards the surface $j_{\mathrm{BER}}(x)$ and being reflected at the surface $(x=0)$ presents the initial rate of transmitting SE at the surface $j_{\mathrm{BET}}(x=0)$, i.e.:

$$
j_{\mathrm{BET}}(x=0)=\sqrt{\frac{\chi}{\bar{E}_{\mathrm{SE}}}} j_{\mathrm{BER}}(x=0) \simeq 0.3 j_{\mathrm{BER}}(x=0)
$$

where $\chi=0.9 \mathrm{eV}$ is the surface electron affinity and $\bar{E}_{\mathrm{SE}}$ the mean kinetic energy of SE in the conduction band of $\mathrm{AL}_{2} \mathrm{O}_{3}$ or $\mathrm{SiO}_{2}$, see Refs. 40,19 and 20. The part of SE non-reflected at the surface is emitted into the vacuum presenting the current $j_{\mathrm{SEE}}$ and the rate of SE emission $\sigma=j_{\mathrm{SEE}} / j_{0}$ :

$$
j_{\mathrm{SEE}}=\left(1-\sqrt{\frac{\chi}{\bar{E}_{\mathrm{SE}}}}\right) j_{\mathrm{BER}}(x=0) \simeq 0.7 j_{\mathrm{BER}}(x=0)
$$

The SE emission-depth dependence (origin where SE are coming from) is given by the SE reverse consecutive transport:

$$
j_{\mathrm{SEE}}(x)=j_{\mathrm{SEE}}(x-\Delta x) \cdot W_{\mathrm{EFR}}
$$


starting at the surface with the emission current $j_{\mathrm{SEE}}(0)$ of Eq. (15). These emitted SE extracted from depth $x$, of course, affect the charge balance in the depth $x$ and will not contribute to the subsequent electron drift current $j$ DER . Thus we have to substract in the forthcoming drift equation Eq. (18) the part of created and finally emitted SE from the depth $x$ :

$$
g_{\mathrm{SEE}}=j_{\mathrm{SEE}}(x)\left(1-W_{\mathrm{EFR}}\right)
$$

because this part is taken off from the overall charge balance. That is new with respect to Refs. 19 and 20. Of course, the drifting hole current $j_{\mathrm{DHT}}^{\mathrm{DHR}}(x)$ of Eq. (19) will not be affected.

\section{Drift currents with trapping and recombination}

Ballistic electrons and holes are scattered over field-dependent attenuation lengths $\lambda_{\mathrm{E}}(F)$ and $\lambda_{\mathrm{H}}(F)$, respectively, as given by Eqs. (10) - (13). As an extension to our previous work Refs. 19 and 20 they continue their motion by drift and diffusion until they will recombine or they will be trapped, see the main scheme in Fig. 5.

Thus we have a convection term from the neighbouring adjacent cell $x \pm \Delta x$ as well as sources (generation) of drift carriers from the attenuated and exhausted, i.e. absorbed ballistic electrons and holes: $j_{\mathrm{BE}}(x)\left(1-W_{\mathrm{EF}}(x)\right)$ and $j_{\mathrm{BH}}(x)\left(1-W_{\mathrm{HF}}(x)\right)$, respectively, given by Eqs. (12) and (13). On the other hand, in Eq. (18) we have to take off the vacuumemitted SE rate: $j_{\mathrm{SEE}}(x)\left(1-W_{\mathrm{EFR}}(x)\right)$ from the charge balance in then depth $x$ as given by Eq. (17). Holes are not emitted into vacuum and so they appear only with their ballistic attenuation source in Eq. (19).

Thus we may write for drifting (D) electrons (E) in reverse (R) and transmission $(\mathrm{T})$ 
directions:

$$
\begin{aligned}
& j \mathrm{DET}(x)=\left\{j_{\mathrm{DET}}^{\mathrm{DER}}(x \pm \Delta x)+j_{\mathrm{BET}}^{\mathrm{BER}}(x)\left[1-W_{\mathrm{EFT}}^{\mathrm{EFR}}(x)\right]-\frac{1}{2} j_{\mathrm{SEE}}(x)\left[1-W^{\mathrm{EFR}}(x)\right]\right\} \times \\
& \times \underbrace{\exp \left[-\frac{\varrho_{H 1}}{e_{0}} S_{\mathrm{EH} 1} \Delta x\right]}_{W_{\mathrm{EH} 1}} \cdot \underbrace{\exp \left[-\frac{\varrho_{H 2}}{e_{0}} S_{\mathrm{EH} 2} \Delta x\right]}_{W_{\mathrm{EH} 2}} \cdot \underbrace{\exp \left[-\left(N_{1}-\frac{\varrho_{E 1}}{e_{0}}\right) S_{\mathrm{E} 1} \Delta x\right]}_{W_{\mathrm{E} 1}} \cdot \underbrace{\exp \left[-\left(N_{2}-\frac{\varrho_{E 2}}{e_{0}}\right) S_{\mathrm{E} 2} \Delta x\right]}_{W_{\mathrm{E} 2}}
\end{aligned}
$$

and for drifting holes $(\mathrm{H})$, respectively:

$$
\begin{aligned}
& j_{\mathrm{DHT}}^{\mathrm{DHR}}(x)=\left\{j_{\mathrm{DHT}}^{\mathrm{DHR}}(x \pm \Delta x)+j_{\mathrm{BHT}}^{\mathrm{BHR}}(x)\left[1-W^{\mathrm{HFR}}(x)\right]\right\} \times \\
& \times \underbrace{\exp \left[-\frac{\varrho_{E 1}}{e_{0}} S_{\mathrm{HE} 1} \Delta x\right]}_{W_{\mathrm{HE} 1}} \cdot \underbrace{\exp \left[-\frac{\varrho_{E 2}}{e_{0}} S_{\mathrm{HE} 2} \Delta x\right]}_{W_{\mathrm{HE} 2}} \cdot \underbrace{\exp \left[-\left(H_{1}-\frac{\varrho_{H 1}}{e_{0}}\right) S_{\mathrm{H} 1} \Delta x\right]}_{W_{\mathrm{H} 1}} \cdot \underbrace{\exp \left[-\left(H_{2}-\frac{\varrho_{H}}{e_{0}}\right) S_{\mathrm{H} 2} \Delta x\right]}_{W_{\mathrm{H} 2}}
\end{aligned}
$$

The recombination probabilities $W_{\mathrm{EH}}$ for electrons with trapped holes $\rho_{H}$ and $W_{\mathrm{HE}}$ for holes with trapped electrons $\rho_{E}$ include the cross sections for these processes $S_{\mathrm{EH} 1}, S_{\mathrm{EH} 2}, S_{\mathrm{HE} 1}$, and $S_{\mathrm{EH} 1}$. For the traping probabilities of electrons in traps with concentration $N_{1}$ and $N_{2}$ and holes in $H_{1}$ and $H_{2}$ we use similar expressions with trap capture cross sections $S_{\mathrm{E} 1}, S_{\mathrm{E} 2}, S_{\mathrm{H} 1}, S_{\mathrm{H} 2}$, respectively. However, we have to use their temperature and field dependences, proposed in the following part.

\section{Capture Cross Sections with Poole-Frenkel Effect}

The free carrier $n$ trapping and release over time $t$ can be expressed by the following "first order" kinetics:

$$
\frac{d n}{d t}=-n v_{\mathrm{D}} \cdot S\left(N-\frac{\varrho}{e_{0}}\right)+\frac{\varrho}{e_{0}} \cdot f \cdot \exp \left(-\frac{E_{T}-\Delta E_{\mathrm{PF}}}{k T}\right)
$$

where $\left(v_{\mathrm{D}} \cdot S\right)$ is the combined trapping rate of the drift velocity $v_{\mathrm{D}}$ and the capture cross section $S$ of traps with concentration $N$, filled by charges $\varrho / e_{0}$ and possessing a detrapping 
probability according to the Poole-Frenkel effect ${ }^{41,42}$ including the escape frequency factor $f$ and a lowering of the thermal activation energy $E_{T}$ by the field-dependent part $\Delta E_{\mathrm{PF}}$. Substitution of time by $d t=d x / v_{\mathrm{D}}$ and taking the non-occupied trapping sites $\left(N-\varrho / e_{0}\right)$ and the capture cross section $S$ in front of the paranthesis we get:

$$
\frac{d n}{n}=-\left(N-\frac{\varrho}{e_{0}}\right) S\left[1-\frac{\varrho / e_{0}}{n v_{\mathrm{D}} \cdot S\left(N-\frac{\varrho}{e_{0}}\right)} \cdot f \cdot \exp \left(-\frac{E_{T}-\Delta E_{\mathrm{PF}}}{k T}\right)\right] d x
$$

with an effective capture cross section modulation given in the brackets and changing between: 0 and 1 . The exponential factor in the brackets will dominate strongly the PooleFrenkel term. Thus, let us introduce an approximation and simplification of the complicated modulation factor of Eq. (21) as follows:

$$
\frac{d n}{n}=-\left(N-\frac{\varrho}{e_{0}}\right) S_{0}\left[1-\exp \left(-\frac{E_{T}-\Delta E_{\mathrm{PF}}}{k T}\right)\right] d x
$$

Here, trapping as well as de-trapping are simultaneously included in a Poole-Frenkel modified cross section and with that we get again the most common temperature-dependent firstorder kinetics. As a matter of self, the trapping probability changes between zero for high temperatures and the full cross section $S_{0}$ for low temperatures.

On the other hand, the Poole-Frenkel activation energy is decreased by an electric field $F$, given by:

$$
\begin{gathered}
\Delta E_{\mathrm{PF}}=2 \frac{e^{3 / 2}}{\left(4 \pi \varepsilon_{0} \varepsilon_{r}\right)^{1 / 2}} F^{1 / 2}=\beta_{\mathrm{PF}} F^{1 / 2} \\
\frac{\Delta E_{\mathrm{PF}}}{\mathrm{eV}}=10^{4}\left(\frac{e}{\pi \varepsilon_{0} \varepsilon_{r}}\right)^{1 / 2} F_{\frac{\mathrm{MV}}{\mathrm{cm}}}^{1 / 2} \simeq 0.24 \mathrm{eV} \cdot F_{\frac{\mathrm{MV}}{\mathrm{cm}}}^{1 / 2} \quad \text { for } \mathrm{Al}_{2} \mathrm{O}_{3} ; \quad \varepsilon_{r}=10
\end{gathered}
$$

Thus we get a Poole-Frenkel modified capture cross section

$$
S_{\mathrm{PF}}=S_{0}\left[1-\exp \left(-\frac{E_{T}-\Delta E_{\mathrm{PF}}}{k T}\right)\right]
$$

approaching $S_{\mathrm{PF}} \rightarrow S_{0}$ for low temperatures $T \rightarrow 0$ and low fields $F \rightarrow 0$ and vanishing $S_{\mathrm{PF}} \longrightarrow 0$ for high temperatures and high electric fields as demonstrated in Fig. 6. A proof 
of the correspondence of the relations Eqs. (20) - (22) will be given in a forthcoming paper.

Now we may rewrite the trapping probabilities in Eqs. (18) and (19) by means of the effective trapping-detrapping probabilities of Eq. (25):

$$
\begin{aligned}
& W_{\mathrm{E} 1}=\exp \left\{-\left(N_{1}-\frac{\varrho_{\mathrm{E} 1}}{e_{0}}\right) S_{\mathrm{E} 10}\left[1-\exp \left(-\frac{E_{\mathrm{E} 1}-\Delta E_{\mathrm{PF}}}{k T}\right)\right] \Delta x\right\} \\
& W_{\mathrm{E} 2}=\exp \left\{-\left(N_{2}-\frac{\varrho_{\mathrm{E} 2}}{e_{0}}\right) S_{\mathrm{E} 20}\left[1-\exp \left(-\frac{E_{\mathrm{E} 2}-\Delta E_{\mathrm{PF}}}{k T}\right)\right] \Delta x\right\} \\
& W_{\mathrm{H} 1}=\exp \left\{-\left(H_{1}-\frac{\varrho_{\mathrm{H} 1}}{e_{0}}\right) S_{\mathrm{H} 10}\left[1-\exp \left(-\frac{E_{\mathrm{H} 1}-\Delta E_{\mathrm{PF}}}{k T}\right)\right] \Delta x\right\} \\
& W_{\mathrm{H} 2}=\exp \left\{-\left(H_{2}-\frac{\varrho_{\mathrm{H} 2}}{e_{0}}\right) S_{\mathrm{H} 20}\left[1-\exp \left(-\frac{E_{\mathrm{H} 2}-\Delta E_{\mathrm{PF}}}{k T}\right)\right] \Delta x\right\}
\end{aligned}
$$

These capture cross sections and respective trapping probabilities unify the trapping and de-trapping process by means of a Poole-Frenkel modified net trapping rate according to Eq. (25).

\section{E. Trapped charges and recombination}

In order to get the trapped and non-passing charges over the distance $\Delta x$ during the time interval $\Delta t$ we use the probabilities of non-passing currents: $(1-W)$ according to Eqs. (18) and (19) and Eqs. (26) - (29) :

$$
\begin{aligned}
& \Delta \varrho_{\mathrm{E} 1}=\left[\frac{j_{\mathrm{DER}}(x)+j_{\mathrm{DET}}(x)}{\Delta x}\left(1-W_{\mathrm{E} 1}(x)\right)-\frac{j_{\mathrm{DHR}}(x)+j_{\mathrm{DHT}}(x)}{\Delta x}\left(1-W_{\mathrm{HE} 1}(x)\right)\right] \cdot \Delta t \\
& \Delta \varrho_{\mathrm{E} 2}=\left[\frac{j_{\mathrm{DER}}(x)+j_{\mathrm{DET}}(x)}{\Delta x}\left(1-W_{\mathrm{E} 2}(x)\right)-\frac{j_{\mathrm{DHR}}(x)+j_{\mathrm{DHT}}(x)}{\Delta x}\left(1-W_{\mathrm{HE} 2}(x)\right)\right] \cdot \Delta t \\
& \Delta \varrho_{\mathrm{H} 1}=\left[\frac{j_{\mathrm{DHR}}(x)+j_{\mathrm{DHT}}(x)}{\Delta x}\left(1-W_{\mathrm{H} 1}(x)\right)-\frac{j_{\mathrm{DER}}(x)+j_{\mathrm{DET}}(x)}{\Delta x}\left(1-W_{\mathrm{EH} 1}(x)\right)\right] \cdot \Delta t \\
& \Delta \varrho_{\mathrm{H} 2}=\left[\frac{j_{\mathrm{DHR}}(x)+j_{\mathrm{DHT}}(x)}{\Delta x}\left(1-W_{\mathrm{H} 2}(x)\right)-\frac{j_{\mathrm{DER}}(x)+j_{\mathrm{DET}}(x)}{\Delta x}\left(1-W_{\mathrm{EH} 2}(x)\right)\right] \cdot \Delta t
\end{aligned}
$$

However, we would count too much charges because of dropping currents $j(t)$ to $j(t+\Delta t)$ during each $\Delta t$ cycle. Thus we have to re-normalize the trapped charges with regard to 
the real current drains and sources according to the general transport equation: Eq. (1) numerically performed here by a three-point derivative:

$$
\Delta \varrho_{\mathrm{tot}}=-\frac{j_{\mathrm{tot}}(x+\Delta x)-j_{\mathrm{tot}}(x-\Delta x)}{2 \Delta x} \Delta t
$$

The total current is given by summation of all currents with their real signs:

$$
j_{\mathrm{tot}}(x)=-j_{\mathrm{PE}}+j_{\mathrm{BER}}-j_{\mathrm{BET}}-j_{\mathrm{BHR}}+j_{\mathrm{BHT}}+j_{\mathrm{DER}}-j_{\mathrm{DET}}-j_{\mathrm{DHR}}+j_{\mathrm{DHT}}
$$

Of course, the secondary emission current $j_{\mathrm{SEE}}(x)$ of Eq. (16) is already included in $j_{\mathrm{BER}}(x)$. Now, with Eq. (34) we get the real resulting net charge $\rho_{\text {tot }}$ due to the total current flux including all trapping and recombination and we have to renormalize the trapped charges in Eqs. (30) - (33) with respect to $\rho_{\text {tot }}$. In case the real net charge balance is negative:

$$
\Delta \varrho_{\text {tot }} \leq 0
$$

we have to calculate the trapped electron charges from Eqs. (30) and (31):

$$
\begin{aligned}
& \Delta \varrho_{\mathrm{E} 1}=\frac{\Delta \varrho_{\mathrm{E} 1}}{\Delta \varrho_{\mathrm{E} 1}+\Delta \varrho_{\mathrm{E} 2}} \cdot\left|\Delta \varrho_{\mathrm{tot}}\right| \\
& \Delta \varrho_{\mathrm{E} 2}=\frac{\Delta \varrho_{\mathrm{E} 2}}{\Delta \varrho_{\mathrm{E} 1}+\Delta \varrho_{\mathrm{E} 2}} \cdot\left|\Delta \varrho_{\mathrm{tot}}\right|
\end{aligned}
$$

whereas the trapped hole charges remain unchanged: $\Delta \varrho_{\mathrm{H} 1}=0$ and $\Delta \varrho_{\mathrm{H} 2}=0$.

In case of a positive net charge:

$$
\Delta \varrho_{\text {tot }}>0
$$

we have the renormalize the trapped hole charges of Eqs. (32) and (33):

$$
\begin{aligned}
& \Delta \varrho_{\mathrm{H} 1}=\frac{\Delta \varrho_{\mathrm{H} 1}}{\Delta \varrho_{\mathrm{H} 1}+\Delta \varrho_{\mathrm{H} 2}} \cdot\left|\Delta \varrho_{\mathrm{tot}}\right| \\
& \Delta \varrho_{\mathrm{H} 2}=\frac{\Delta \varrho_{\mathrm{H} 2}}{\Delta \varrho_{\mathrm{H} 1}+\Delta \varrho_{\mathrm{H} 2}} \cdot\left|\Delta \varrho_{\mathrm{tot}}\right|
\end{aligned}
$$


and the trapped electron charges remain unchanged: $\Delta \varrho_{\mathrm{E} 1}=0$ and $\Delta \varrho_{\mathrm{E} 2}=0$.

Of course, the total charge $\varrho_{\text {tot }}$ of traps should be consistent with that of current divergences, Eqs. (1) and (34), i.e.

$$
\varrho_{\text {tot }}(x)=-\varrho_{\mathrm{E} 1}-\varrho_{\mathrm{E} 2}+\varrho_{\mathrm{H} 1}+\varrho_{\mathrm{H} 2}
$$

From this total charge distribution $\rho_{\text {tot }}(x, t)$ the resulting electric field $F(x, t)$ and potential $V(x, t)$ distributions are obtained by respective intergrations shown in Fig. 1. The potential value at the surface $V(x=0, t)=V_{0}(t)$ represents the resulting surface potential $V_{0}$ due to electron beam irradiation, secondary electron emission, and selfconsistent charging. The whole computation procedure is schematically shown in Fig. 7.

\section{F. Retarding field effects to SE emission}

In case that the floating surface potential $V_{0}$ is greater (more positive) than the potential $V_{\mathrm{G}}$ of the first electrode in the vacuum in front of the surface (grid, chamber wall etc.), emitted SE will be retarded and will return to their origin "positive" spot of emission. As we have seen by Eq. (15) the SE emission is given by: $J_{\mathrm{SEE}}=0.7 j_{\mathrm{BER}}(x=0)$. The energy distribution of emitted SE is commonly non-material-dependent and possesses a mean energy of $\bar{E}_{\mathrm{SEE}} \simeq 5 \mathrm{eV}$ in the vacuum. Assuming a nearly exponential energy distribution a negative retarding grid electrode $V_{\mathrm{G}}<V_{0}$ will diminish the SEE rate ${ }^{17-20}$ to:

$$
j_{\mathrm{SEE}}=0.7 j_{\mathrm{BER}}(x=0) \exp \left(-\frac{V_{0}-V_{\mathrm{G}}}{5 \mathrm{Volt}}\right)
$$

and, consequently, the rate of returning tertiary electrons (TE) is

$$
j_{\mathrm{TE}}=0.7 j_{\mathrm{BER}}(x=0)\left[1-\exp \left(-\frac{V_{0}-V_{\mathrm{G}}}{5 \mathrm{Volt}}\right)\right] .
$$


Thus the rate of ballistic electrons starting from the surface $(x=0)$ in transmission $(\mathrm{T})$ direction is increased by the returning TE:

$$
j_{\mathrm{BET}}(x=0)=0.3 j_{\mathrm{BER}}(x=0)+j_{\mathrm{TE}}\left(V_{0}>V_{\mathrm{G}}\right)
$$

and with Eq. (44) we get:

$$
j_{\mathrm{BET}}(x=0)=j_{\mathrm{BER}}(x=0)\left[1-0.7 \exp \left(-\frac{V_{0}-V_{\mathrm{G}}}{5 \text { Volt }}\right)\right]
$$

Thus, for $V_{0}>V_{\mathrm{G}}$, Eqs. (14) and (15) have to be replaced by Eqs. (43) and (46), respectively. Otherwise, for $V_{0} \leq V_{\mathrm{G}}$, the former Eqs. (14) and (15) remain unchanged.

Last but not least, the actual secondary electron emission rate $\sigma$ is given by

$$
\sigma(t)=\eta+\delta(t)=1+\frac{j(x=0, t)}{j_{0}}
$$

including the fraction $\eta$ of backscattered PE and the true SE released from the target material with the rate $\sigma(t)$ changing with time due to the charging of non-conductive targets.

\section{RESULTS of SELFCONSISTENT CHARGING}

As already mentioned in the previous part, the computation scheme is presented in Fig. 7. The first simulations have been performed for $3 \mathrm{~mm}$ thick (bulk) alumina samples by means of material parameters mostly given already in our former work of Ref. 20 (Tab.1); others are described already in the respective text parts of this paper. In order to protect the samples from thermal irradiation effects and material modifications, we used in our measurements as well as simulations a slightly "defocused" electron beam of $100 \mathrm{nA}$ impinging an area of $1 \mathrm{~mm}^{2}$ leading to a primary electron current density of $j_{0}=10^{-5} \mathrm{~A} / \mathrm{cm}^{2}$. This planar geometry of $1000 \mu \mathrm{m}$ beam width versus only $3 \mu \mathrm{m}$ irradiation depth maintains 
the assumption of the planar 1-dimensional target model. So we may use mean ballistic attenuation lengths for secondary electrons $\lambda_{E, 0}=5 \mathrm{~nm}$, for holes $\lambda_{H, 0}=2 \mathrm{~nm}$ and respective attenuation field factors $\beta_{E}=4.6 \mathrm{~cm} / \mathrm{MV}$ and $\beta_{H}=0.8 \mathrm{~cm} / \mathrm{MV}$ in accordance with Refs. 20 - 22. More difficult is the selection of appropriate electron and hole trap concentrations $N, H$, their capture cross sections $S_{E}, S_{H}$, their thermal activation energies $E_{E}, E_{H}$, and recombination cross sections $S_{E H}$ and $S_{H E}$, as given in Fig. 5. For our first calculations presented here we choose the following data, estimated from Refs. 43 - 48: shallow trap concentrations $N_{E 1}=N_{H 1}=10^{20} \mathrm{~cm}^{-3}$ corresponding to high concentrations of selftrapping by small polaron formation; their capture cross sections $S_{E 1}=S_{H 1}=10^{-13} \mathrm{~cm}^{2}$ and thermal activation energies $E_{E 1}=E_{H 1}=0.2 \mathrm{eV}$. Of course, the deeper traps possess less concentrations: $N_{E 2}=N_{H 2}=10^{18} \mathrm{~cm}^{-3}, S_{E 2}=S_{H 2}=10^{-13} \mathrm{~cm}^{2}$, and $E_{E 2}=E_{H 2}=2 \mathrm{eV}$. The Coulomb-attractive recombination can be described by a relatively high cross section $S_{E H}=S_{H E}=10^{-11} \mathrm{~cm}^{2}$. We should mention here that a more diversive trap parameter selection and optimalization will be done when direct comparison with experimental data will be undertaken in the next future.

\section{$\underline{\text { A. Charging of open samples }}$}

These samples possess an open, non-covered surface and the secondary electron emission is only limited by the height of the surface barrier, i.e. by the electron affinity $\chi_{\mathrm{Al}_{2} \mathrm{O}_{3}}=0.9 \mathrm{eV}$, as described in chapt.II.B. Of course, all holes and also low energy drifting electrons will be totally reflected (chapt.II.C). The total current $j_{\text {tot }}(x)$, the charge $\rho(x)$ and field $F(x)$ distributions in dependence on electron beam irradiation time $t=(10 \ldots 100) \mathrm{ms}\left(E_{0}=20 \mathrm{keV}\right.$, $j_{0}=10^{-5} \mathrm{~A} / \mathrm{cm}^{2}$ ) are presented in Fig. 8. On the left hand side the distributions are given 
in a zoomed nanometer scale beneath the surface where especially the emerging secondary electrons are coming from. Here we see the built-up of a positive charge distribution with a center of gravity at about $2.5 \mathrm{~nm}$. The field is increasing positively enforcing field-enhanced secondary electron emission into the vacuum. On the right hand side of Fig. 8 we see the respective bulk distributions. The field remains positive up to $0.5 \mu \mathrm{m}$ sweeping electrons towards the surface and holes into the bulk. Then, beyond $0.5 \mu \mathrm{m}$, the field changes to negative values and keeping almost constant up to the support electrode at $x=d=3 \mathrm{~mm}$. The drifting and finally trapped charges form a fourfold charge distribution: plus-minusplus-minus. The positive surface charge is due to emitted SE and remaining holes as mentioned already in context with the zoomed left hand side of Fig. 8. Then the positive field is separating electrons towards the surface (negative) and holes into the bulk (positive). Finally, because of a greater ballistic allenuation length $\lambda_{E}$ electrons are straggling into deeper regions than holes and forming the negative deep charges. Moreover, because of the field change to negative values, drifting electrons are swept into the deep regions. Thus we get a plus-minus-plus-minus charge distributions as already observed in the former only ballistic model of Ref. 20 .

Here we should mention, that all distributions, especially of $j_{\text {tot }}(x)$ and $\rho_{\text {tot }}(x)$ are shrinked towards the surface and do not reach the electron maximum range $R\left(E_{0}\right)$ in a remarkable extend. The reason for that is given by the overall negative charging and the resulting negative surface potential $V_{0}$ as we see in Fig. 9. After $10 \mathrm{~ms}$ irradiation it approaches already $-13 \mathrm{keV}$ and the resulting initial energy of the incident electron beam is $E_{0}^{\prime}=E_{0}+e V_{S}=7 \mathrm{keV}$. Thus the distributions are shrinked to about $R\left(E_{0}^{\prime}\right) \approx 0.6 \mu \mathrm{m}$ instead extended to the maximum range $R\left(E_{0}=30 \mathrm{keV}\right) \approx 3 \mu \mathrm{m}$ for non-charged samples. Looking to the time dependences in Fig. 9 we see an increasing secondary electron emission 
rate from the initial value $\sigma_{\mathrm{SEE}}(t=0)=\sigma_{0}<1$ to the stationary final state of $\sigma_{\mathrm{SEE}}=1$ for thick bulk samples within tens of ms. Thus the SEE is enhanced for high electron beam energies due to the built-up of a positive field over the SE escape region. However, as to be seen in Fig. 9 (top) the SE rate tends to overflow the $\sigma=1$ value by about $\Delta \sigma=+0.004$, but only for a short time of $\Delta t \approx 20 \mathrm{~ms}$. It corresponds to a small additional positive charging in the SE escape zone $\Delta x \approx 10 \mathrm{~nm}$ of $\Delta \rho=j_{0} \cdot \Delta \sigma \cdot \Delta t / \Delta x \approx 10^{-3} \mathrm{As} / \mathrm{cm}^{3}$ which is relaxes within time $\Delta t$. Comparing this excessive charge $\Delta \rho$ with the overall distribution $\rho(x)$ in Fig. 8 we estimate only to $1 \Delta \rho / \rho \approx 0.001$. These small charge fluctuation and redistribution are due to the inertia of the time-dependent trapping and de-trapping processes. A similar behavior is reported by Bragga, Blaise et al. ${ }^{47}$ who observed such overflows $\sigma>1$ for very high incident current densities $j_{0}$, very probably, associated with inertia effects of trapping-detrapping too.

The latter processes are also responsible for the slight decrease of the negative surface potential $V_{0}$ in the saturation region, Fig. 9 (bottom). However, this saturation behavior is not pure exponentially as described recently in Ref. 48. We may argue that the well-known exponential charging behavior of a simple capacitor: $d Q=I(t) d t$ cannot be taken over for electron beam charging because the current $I(t)$ in a capacitor is decreasing exponentially during charging whereas the electron beam and its penetration depth $R\left(E_{0}^{\prime}\right)$ follow other relations as shown in part II A. Hence the electron beam forces the charges to penetrate longer times to a depth $x$ it will fill the insulator faster than a respective capacitor is commonly loaded.

This is also the main reason that capacitor-like double-layer charge models, as discussed in the 1. Introduction, become very questionable.

Nevertheless, the negative volume charge is exceeding the positive surface charge and the 
resulting-in surface potential $V_{0}=V(x=0)$ is approaching high negative values: increasing with the electron beam energy $E_{0}=10 \mathrm{keV}$ and $V_{0}=-4 \mathrm{kV}$ up to $E_{0}=30 \mathrm{keV}$ and $V_{0}=-24 \mathrm{kV}$ as expected. However, we should mention that these negative surface potential values obtained by the new Flight-Drift Model FDM are higher (more negative) that the former ones of the only ballistic flight model of Ref. 20. The reason is the stronger charge separation by the drift transport and the deeper sweeping-in of drifting electrons into the bulk volume.

A certain interest bears the SEE transition region $(\stackrel{\gtrless}{<})$ from $\sigma_{\mathrm{SEE}}\left(E_{0}<E_{0}^{I I}\right)>1$ to $\sigma_{\mathrm{SEE}}\left(E_{0}>E_{0}^{I I}\right)<1$ at $E_{0}=E_{0}^{I I} \simeq 4.2 \mathrm{keV}$, see Figs. 10 and 11 . There for higher beam energies $E_{0} \geq 5 \mathrm{keV}$ the SE rates $\sigma_{\mathrm{SEE}}$ increases from initial values $\sigma_{0}<1$ to the final stationary value $\sigma_{\mathrm{SEE}}=1$ within milliseconds as we have already observed for high energies in Fig. 9. The respective surface potential $V_{0}(t)$ in Fig. 11 becomes negative, shows a turnaround when $\sigma_{\mathrm{SEE}}(t) \rightarrow 1$, growing slightly and approaches finally constant negative values; for $E_{0}=5 \mathrm{keV}$ even $V_{0}$ approaches $V_{G}=0$ Volt. Going further downwards to $E_{0}=4 \mathrm{keV}$ we observe the transition region $\sigma_{0}\left(E_{0}^{I I} \approx 4.2 \mathrm{keV}\right)=1$ and with still decreasing beam energies $E_{0} \leq 4 \mathrm{keV}$ the initial SEE rate $\sigma_{0}$ becomes greater unit; $\sigma_{0}>1$, and the resulting charging is positive with positive surface potentials $V_{0}>0$. However, the positive charging-up and the increase of the $\mathrm{SE}$ rate $\sigma_{\mathrm{SEE}}>1$ are rapidly stopped by the grounded grid $V_{G}=0$ and the retarding field set up between the positive surface $V_{0}>0$ and the grounded grid $V_{G}=0$ as described in chapt.II.F and Eqs. (43) and (44). This retarding field process occurs within some nanoseconds. Later on, the surface potential remains nearly constant at slightly positive values, however, with some fluctuations due to long term drift processes and charge re-distributions. All these phenomena are in agreement with experimental observations, see Refs. 17, 18 and 49, 50. 


\section{B. Ion-covered ESEM sample}

In order to avoid strong negative charging of bulk insulating samples during electron beam irradiation, e.g. in scanning electron microscopy (SEM), the samples will be coated by a thin conduction layer, e.g. by a metal or carbon layer, or the electron beam probing is performed in an environmental scanning electron microscope ESEM. The latter one uses an environmental gas, mostly water vapor, of low pressure (some Torr) which will be ionized by the electron beam and by the backscattered (BE) and secondary (SE) electrons. Even the BE and SE signals are amplified by gas discharge and avalanching processes in the low pressure gas environment. On the other hand, positive gas ions are attracted by the negatively charged sample surface and will compensate a certain part of negative charges. It leads to a dynamical equillibrium of charging-discharging at the surface and a resulting less negative surface potential of only about $V_{0}=-500$ Volt, calculated and measured e.g. by M. Todt et al. ${ }^{28,29}$. Thus we use this value for our simulation according to Fig. 1, part B. The electron beam energy was also choosen $E_{0}=20 \mathrm{keV}$ as for open samples in Fig. 8 . There the resulting surface potential did approach $V_{0}=-14 \mathrm{kV}$, but here for the ESEM sample it will be fixed at $V_{0}=-0.5 \mathrm{kV}$. Moreover, we have to take regard to a modified surface barrier and surface reflectance of ballistic electrons by the positive ion layer as shown in Fig. 12, part B. As a first approximation we chose the surface reflectance coefficient $b_{i}$ as that of the open, non-covered surface $b_{0}$, i.e. $b_{i}=b_{0}=0.3$.

The results of respective calculations $j_{\text {tot }}(x, t), \rho(x, t)$, and $F(x, t)$ are presented in Fig. 13. For all these distributions we see much less fluctuations of the internal quantities than for the open sample in Fig. 8, however, roughly the same order of magnitude. Of course, as expected, the field beneath the surface with $0.2 \mathrm{MV} / \mathrm{cm}$ is twice of that of the 
open layer. Moreover, it is centered at the surface and has no component into the bulk. The magnitude of the potential distribution is very moderate and does not exceed the ion-fixed surface value $V_{0}=-500$ Volt so much: -525 Volt at the depth of the maximum electron range $x \approx R \approx 3 \mu \mathrm{m}$. Obviously, there is almost no retarding effect to the incident primary electrons: $E_{0}^{\prime}=E_{0}+e V_{0}$ as in open samples. Summarizing all the effects we may state, that in case of ion-covered insulating samples a strong charging is mostly avoided and the spatial electron beam excitation and probing is rather not affected. Thus the intention of the ESEM techniques is well obtained.

\section{Metal-covered and grounded surface}

As already mentioned in context with Figs. 1 and 12 thin metal or conducting material layers, electrically grounded, are conventionally used to avoid surface charging of insulating samples. These layers are mostly evaporated or sputtered onto the surface, their thickness is very thin, about $10 \mathrm{~nm}$, in order not to affect the incident electron beam so much. Nevertheless, as shown in Fig. 12, bottom part C, we have to regard different surface absorption and re-injection conditions for electrons and holes. So we assume that holes and drifting electrons are fully absorbed: $j_{\mathrm{BHT}}(x=0)=j_{\mathrm{DHT}}(x=0)=j_{\mathrm{DET}}(x=0)=0$, whereas excited ballistic SE from the metallic surface layer are injected into the insulator. This part is proportional to the exciting $\mathrm{PE}$ and we may write $j_{\mathrm{BET}}(x=0)=b_{m} \cdot j_{0}$. The internal SE fraction was estimated to $b_{m} \approx 0.5$ almost in accordance to bulk-internal SE measurements ${ }^{51}$.

As we see in Fig. 14 the distributions of currents, charges, fields and potentials are similar to those of the ion-covered and surface potential-fixed layers in Fig. 13. They show 
also much less fluctuations than for open layers in Fig. 8. The plus and minus charges are located near to the surface and the field in thoroughly positive increasing towards the surface to $0.3 \mathrm{MV} / \mathrm{cm}$. The negative potential shows a maximum of only -27 Volt in the depth, nearly at the maximum range $R \approx 3 \mu \mathrm{m}$ of incident electron with energy $E_{0}=20$ $\mathrm{keV}$. Thus the incident and spatially exciting electron beam is not affected by retarding field effects. Thus the main intention of no charging is fullfilled, however, the greatest affect to the SEE measurement, spectroscopy or microscopy is given by the electron scattering in the metallic or conducting material layer on the surface. Therefore, preference should be given to positive ion covered surfaces as discussed in the previous section B in context with ESEM, or even to charge neutralization by "low energy electron rinsing" as commonly used in electron spectroscopy of insulating samples, see e.g. the comprehensive treatment and consideration of L. Reimer ${ }^{52}$.

\section{CONCLUSIONS}

The new flight-drift model (FDM) of selfconsistent electron transport and electrical charge storage in wide-gap insulators reflects a more realistic simulation of these processes in dielectric and insulating materials. Electron-hole creation, their ballistic flight, followed by field-drift transport, and finally trapping in localized states and/or recombination are taken into account. The experimentally accessable quantities of field assisted secondary electron emission $\sigma$ as well as the resulting surface potential $V_{0}$ due to internal current $j(x, t)$, charge $\rho(x, t)$, field $F(x, t)$, and potential $V(x, t)$ distributions are obtained.

The charging of open, i.e. non-covered and floating insulating bulk samples is strongly controlled by the surface potential $V_{0}(x=0)$ and the consequent electron beam retarding 
for negative charging $V_{0}<0$ or even beam accelleration for positive charging $V_{0}>0$ according to the affection of the initial energy $E_{0}^{\prime}=E_{0}+e V_{0}$. Thus the maximum range of incident primary electrons is rapidly diminished for high beam energies $E_{0}>5 \mathrm{keV}$ by negative charging and the internal current, charge and field distributions are shrinked strongly towards the surface.

Contrary to open samples the positive-ion-covered samples of the ESEM techniques possess an only slightly negative surface potential fixed by the positive ions at about $V_{0}=-0.5 \mathrm{kV}$. Here the electron excitation is almost not affected by beam retarding and the charge and field distributions show less fluctuations, however, an increase in magnitude towards the surface.

A similar effect is given in conventional metal or conducting material coated insulating samples. Usually the coating layer is grounded and no electron beam retarding field effects are observed, also within sample where the negative potential distribution does not exceed several tens of Volt, i.e. $V(x)<-50$ Volt. Thus the exciting electron beam is not affected neither in the front of the surface in vacuum nor in the internal bulk insulator. However, one cannot neglect the additional scattering of the incident electron beam as well as of backscattered and secondary electrons within the coating layer.

Thus, in order to prevent charging, the positive-ion-covered surface bears certain advantages versus metal coating.

\section{ACKNOWLEDGEMENT}

We are grateful to G. Damamme for suggesting this work and for fruitful discussions. 


\section{REFERENCES}

1 J. Mannhart, D. G. Schlom, Physik Journal 4, Nr. 6 (June), p. 45, 2005

2 4th International Conference on Electric Charges in Non-Conductive Materials, Le Vide: Science, Techniques et Applications, Vol. Special CSC'4, 2001

3 Symposium on Characterization of Non-conductive and Charging Materials by Microbeam Analysis, Ed. R. Gauvin, August 2002, McGill University Montreal, Canada

4 A. Ibarra, E. R. Hodgson, The ITER project: the role of insulators, Nuclear Instruments and Methods in Physics Research B, 218, 29-35 (2004)

5 V. Griseri, K. Fukunaga, T. Maeno, L. Levy, C. Laurent, D. Payan, IEEE Transaction on Dielectrics and Electrical Insulation, 11, 891-898 (2005)

6 L. Levy, Material Charging, Cedapus Editions 2002, pp. 241-266

7 J. Cazaux, J. of Electron Spectroscopy, 105, 155-185 (1999)

8 M. Belhaj, O. Ibara, M. N. Filippov, E. I. Rau, M. V. Andrianov, Appl. Surf. Sci 177, 58-65 (2001)

9 J. Cazaux, J. Appl. Phys. 89, 8265-8272 (2001)

10 L. Malter, Phys. Rev. 49, 478 (1936)

11 A. Melchinger, S. Hofmann, J. Appl. Phys. 78, 6624 (1995)

12 J. Cazaux, J. Appl. Phys. 85, 1137 (1999)

13 E. Vicario, N. Rosenberg, R. Renoud, Surface and Interface Analysis 22, 115 (1994)

14 J. P. Ganachaud, C. Attard, R. Renoud, Physica Status Solidi (b) 199, 175-184 and 455-465 (1997) 
R. Renoud, F. Mady, J. P. Ganachaud,

J. Phys.: Condensed Matter 14, 231-247 (2002)

16 F. Mady, R. Renoud, C. Attard, J. Bigarré, J.-P. Ganachaud, P. Hourquebie, Eur. Phys. J. Appl. Phys. 20 41-53 (2002)

17 H.-J. Fitting, Habilitation thesis, University of Rostock/Germany, 1978

18 H.-J. Fitting, H. Glaefeke, W. Wild, M. Franke, W. Müller,

Experimentelle Technik der Physik (Berlin) 27, 13-24 (1979)

19 I. A. Glavatskikh, V. S. Kortov, H.-J. Fitting, J. Appl. Phys. 89, 440-448 (2001)

20 X. Meyza, D. Goeuriot, C. Guerret-Piécourt, D. Treheux, H.-J. Fitting,

J. Appl. Phys. 94 5384-5392 (2003)

21 H.-J. Fitting, H. Glaefeke, W. Wild, R. Ulbricht, Experimentelle Technik der Physik (Berlin) 24, 447 (1976)

22 Th. Hingst, M. Hübner, R. Franz, Ch. Kuhr, H.-J. Fitting, Microelectronic Engineering 24, 181 (1994)

23 H.-J. Fitting, J.-U. Friemann, Physica Status Solidi (a) 69, 349 (1982)

24 H.-J. Fitting, J. Boyde, Physica Status Solidi (a) 75, 137 (1983)

25 E. Schreiber, H.-J. Fitting,

J. Electron Spectroscopy \& Related Phenomena 124, 25-37 (2002)

26 H.-J. Fitting, E. Schreiber, I. A. Glavatskikh,

Microscopy \& Microanalysis (USA) 10, 764-770 (2004)

27 X. Meyza, PhD thesis, Ecole Superieure des Mines de Saint-Etienne, 2003

28 M. Toth, M. R. Phillips, B. L. Thiel, A. M. Donald,

J. Appl. Phys. 91, 4479-4491 and 4492-4499 (2002)

29 M. Toth, B. L. Thiel, A. M. Donald, Ultramicroscopy 94 71-87 (2003) 
H.-J. Fitting, H. Glaefeke, W. Wild, Physica Status Solidi (a) 43, 185 (1977)

31 J. E. Holliday, E. J. Sternglass, J. Appl. Phys. 30, 1428 (1959)

32 A. Y. Vyatskin, U. V. Trunev, Radiotekhnika i Elektronika (Moscow), 9, 1899-1905 (1972)

33 J. R. Young, Phys. Rev. 103, 292 (1956)

34 T. E. Everhart, P. H. Hoff, J. Appl. Phys. 42, 5837 (1971)

35 K. Kanaya, S. Okayama, J. Phys. D: Appl. Phys. 5, 43 (1972)

36 H.-J. Fitting, Physica Status Solidi 26, 525 (1974)

37 C. A. Klein, J. Appl. Phys. 39, 2029-2038 (1968)

38 R. C. Alig, S. Bloom, Phys. Rev. Lett. 35, 1522-1525 (1975)

39

H.-J. Fitting, J. Electron Spectroscopy \& Related Phenomena 136, 265-272 (2004)

40 H.-J. Fitting, H. Glaefeke, W. Wild, Surface Sci. 75, 267 (1978)

41 J. Frenkel, Phys. Rev. 54, 647 (1938)

42 J. J. O'Dwyer "Theory of electrical conduction and breakdown in solid dielectrics", Clarendon Press, Oxford 1973

43 T. H. Ning, J. Appl. Phys. 47, 3203 (1976)

44 V. A. J. Van Lint, J. M. Bunch, T. M. Flanagan, Proceedings of the Conference on Radiation Effects and Tritium Technology for Fusion Reactors, ed. by J. S. Watson, F. W. Wiffen, I. L. Bishop, and B. K. Breeden, Gatlinburg, TN (1976), Vol. 11, p. 531

45 P. C. Srivasta, A. Roy Bardhan, D. L. Bhattacharya, Intern. J. Electronics 46, 547-554 (1979)

46 I. I. Milman, V. S. Kortov, S. V. Nikiforov, Radiation Measurements 29, 401-410 (1998) 
D. Bragga, B. Poumellec, V. Cannas, G. Blaise, Y. Ren, M. Christensen,

J. Appl. Phys. 96, 885 (2004)

48 B. Askri, R. Renoud, K. Raouadi, J.-P. Ganachaud,

Europ. Phys. J. Appl. Phys. 32, 29 (2005)

49 H.-J. Fitting and D. Hecht, phys. stat. sol. (a) 108 265-273 (1988)

50 H.-J. Fitting, P. Magdanz, W. Mehnert, D. Hecht, and Th. Hingst, phys. stat. sol. (a) 122 297-309 (1990)

51 H.-J. Fitting, H. Glaefeke, W. Wild, G. Neumann,

J. Phys. D: Appl. Phys. 92499 (1976)

52

L. Reimer, Scanning Electron Microscopy, Physics of Image Formation and

Microanalysis, 2nd Edition, Springer Series in Optical Sciences Vol 45 (1998) chapt. 3.5.2 


\section{Captions of the Figures}

Fig. 1 Plus-minus charge distribution $\rho(x)$ beneath the surface and the respective electrical field $F(x)$ and potential $V(x)$ distributions in a dielectric on a conducting support with an open surface: A; an ion-covered surface: B; and a metal covered and grounded surface: C.

Fig. 2 Measurements (Refs. 31, 32) and experimental fits (Refs. 33 - 36) of the maximum range $R\left(E_{0}\right)$ of electrons with initial energy $E_{0}$ in amorphous $\mathrm{Al}_{2} \mathrm{O}_{3}$.

Fig. 3 Current $j_{\mathrm{PE}}(x)$ of primary electrons with initial energy $E_{0}$ into $\mathrm{Al}_{2} \mathrm{O}_{3}$, their deposition (absorption) function $g_{\mathrm{PE}}(x)$, and their secondary electron generation $g_{\mathrm{SE}}(x)$, according to Eqs. (4) - (9), respectively.

Fig. 4 Mean attenuation lenghts of electrons $\lambda_{\mathrm{EF}}$ and holes $\lambda_{\mathrm{HF}}$ in $\mathrm{Al}_{2} \mathrm{O}_{3}$ as a function of the electric field $F$, according to Eq. (11).

Fig. 5 Scheme of the flight-drift model including the ballistic flight and attenuation followed by drift, trapping, and recombination of electrons and holes.

Fig. 6 Poole-Frenkel-modified capture cross sections $S_{\mathrm{PF}}$ according to Eq. (25) of a shallow trap with thermal activation energy $E_{T}=0.3 \mathrm{eV}$ in dependence on temperature $T$ and electric field $F$. 
Fig. 7 Computation scheme of the selfconsistent charging in insulating samples.

Fig. 8 Total current $j_{\text {tot }}(x)$, charge $\rho(x)$, and field $F(x)$ depth distributions as a function of irradiation time $t$ for an initial beam energy of $E_{0}=20 \mathrm{keV}$ and current density $j_{0}=10^{-5} \mathrm{~A} / \mathrm{cm}^{2}$; left: benath the surface zoomed in $\mathrm{nm}$ scale; right: in the bulk alumina in $\mu \mathrm{m}$ scale. Mention that all the bulk distributions are shrinked towards the surface because of a strong negative charging and respective retarding of the electron beam to $E_{0}^{\prime}=E_{0}-e V_{0}=7 \mathrm{keV}$ with much less electron ranges already after $10 \mathrm{~ms}$ irradiation time.

Fig. 9 Secondary electron emission rate $\sigma$ and surface potenial $V_{0}$ of bulk alumina targets as function of irradiation time $t$ for different electron beam energies $E_{0}: j_{0}=10^{-5} \mathrm{~A} / \mathrm{cm}^{2}$.

Fig. 10 Secondary electron emission rate $\sigma_{\mathrm{SEE}}=\eta+\delta$ in dependence on irradiation time for different $\mathrm{PE}$ energies $E_{0}=(0.5-7) \mathrm{keV}$ in the transition region $\sigma \gtreqless 1$; $j_{0}=10^{-5} \mathrm{~A} / \mathrm{cm}^{2}$.

Fig. 11 Surface potential $V_{0}$ in dependence on irradiation time for different PE energies $E_{0}=(0.5-7) \mathrm{keV}$ in the transition region $\sigma \stackrel{\gtrless}{\gtrless} ; j_{0}=10^{-5} \mathrm{~A} / \mathrm{cm}^{2}$.

Fig. 12 Surface reflection and/or absorption of electrons and holes at: A: open surface; B: positive-ion-covered surface, and C: metallized and grounded surface. 
Fig. 13 Current $j_{\text {tot }}(x)$, charge $\rho(x)$, electric field $F(x)$, and potential $V(x)$ depth distributions beneath the positive-ion-covered surface $\left(V_{0}=-500\right.$ Volt $)$ of alumina in dependence on irradiation time $t$; electron beam energy $E_{0}=20 \mathrm{keV}$ and current density $j_{0}=10^{-5} \mathrm{~A} / \mathrm{cm}^{2}$.

Fig. 14 Current $j_{\text {tot }}(x)$, charge $\rho(x)$, electric field $F(x)$, and potential $V(x)$ depth distributions beneath the positive metal-covered and grounded surface $\left(V_{0}=0\right)$ in dependence on irradiation time $t$, zoomed in $\mathrm{nm}$ scale (left) and presented in $\mu \mathrm{m}$ scale (right); electron beam energy $E_{0}=20 \mathrm{keV}$ and current density $j_{0}=10^{-5} \mathrm{~A} / \mathrm{cm}^{2}$. 


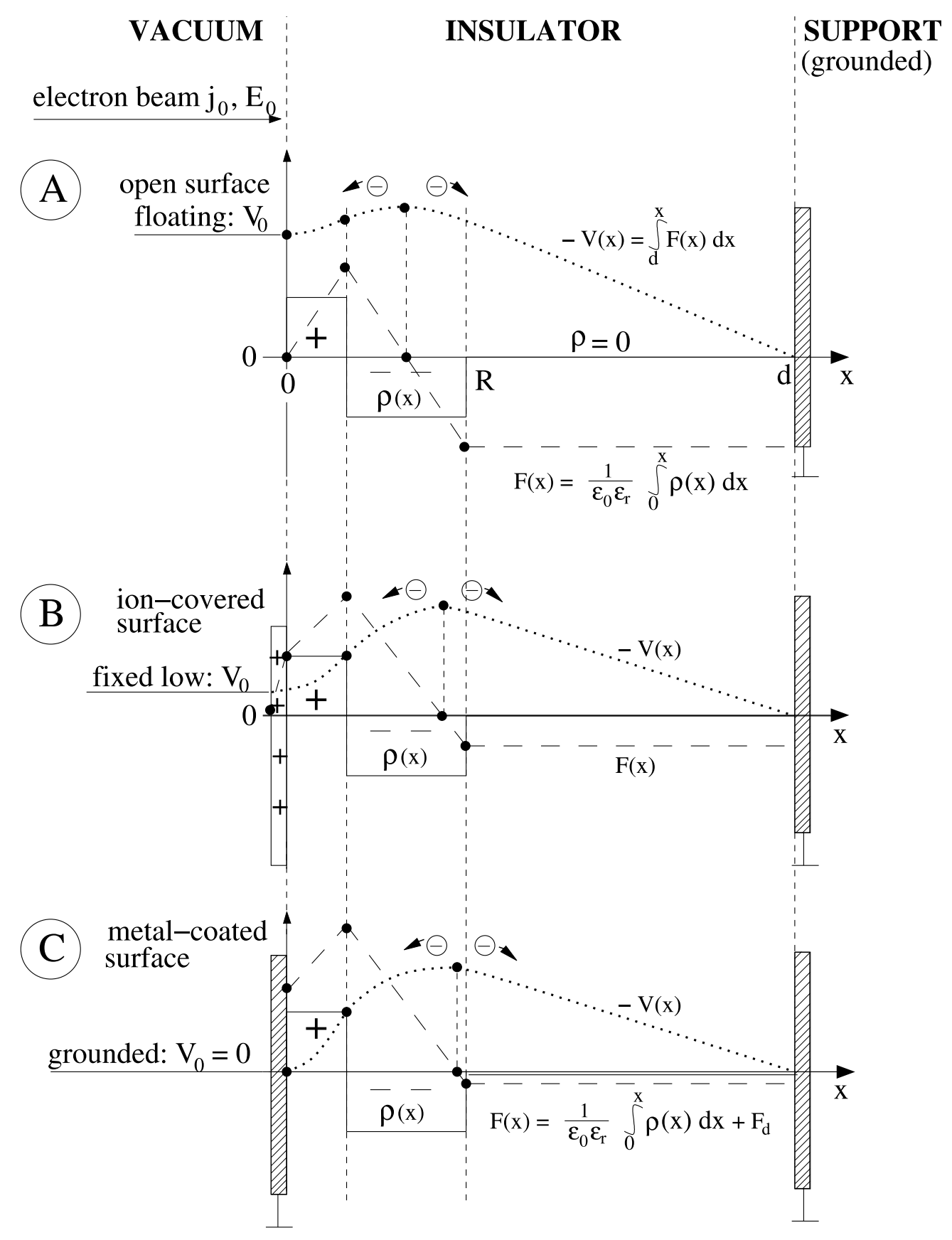

Fig. 1 Plus-minus charge distribution $\rho(x)$ beneath the surface and the respective electrical field $F(x)$ and potential $V(x)$ distributions in a dielectric on a conducting support with an open surface: A; an ion-covered surface: B; and a metal covered and grounded surface: C. 


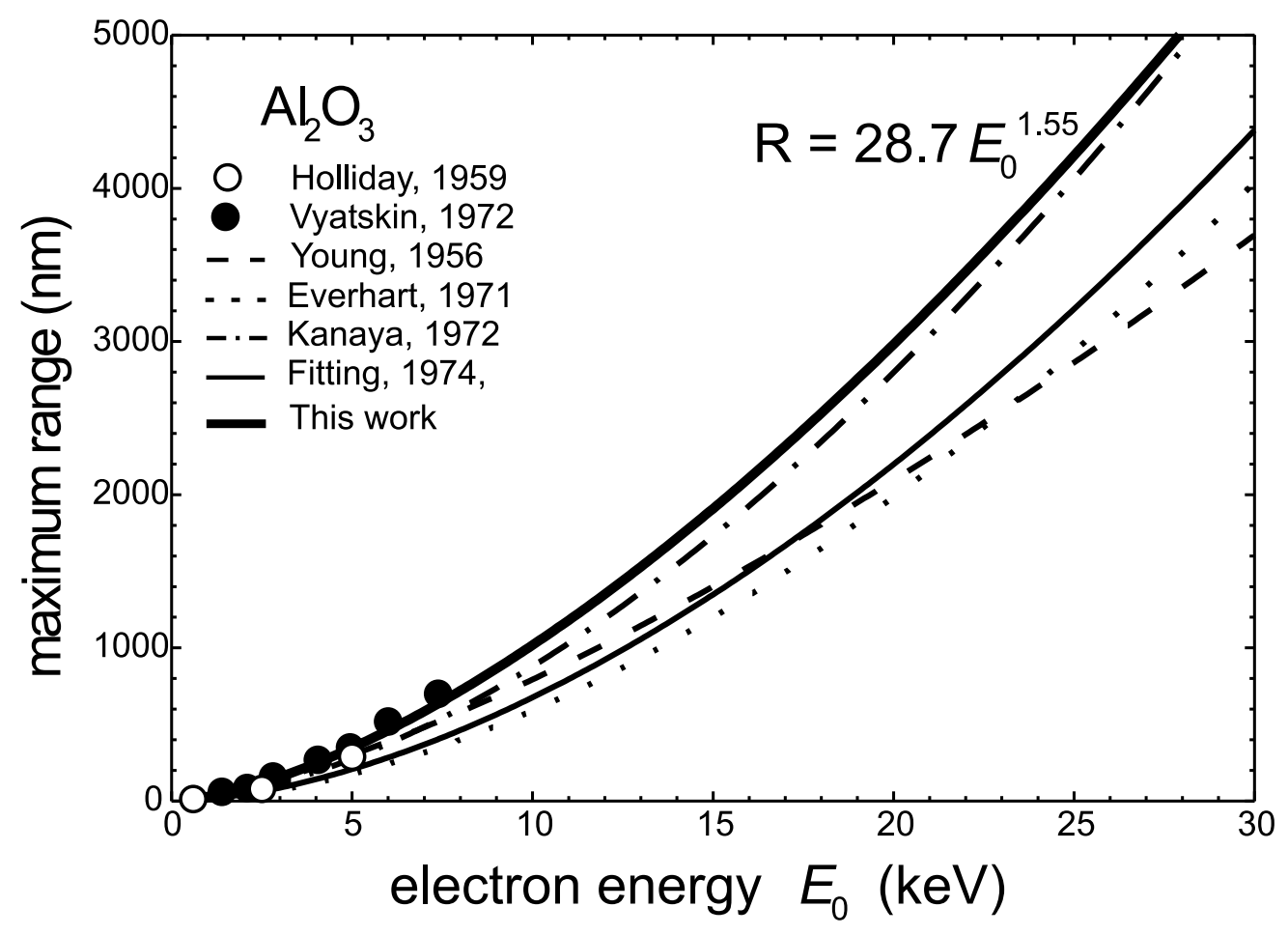

Fig. 2 Measurements (Refs. 31, 32) and experimental fits (Refs. 33 - 36) of the maximum range $R\left(E_{0}\right)$ of electrons with initial energy $E_{0}$ in amorphous $\mathrm{Al}_{2} \mathrm{O}_{3}$. 

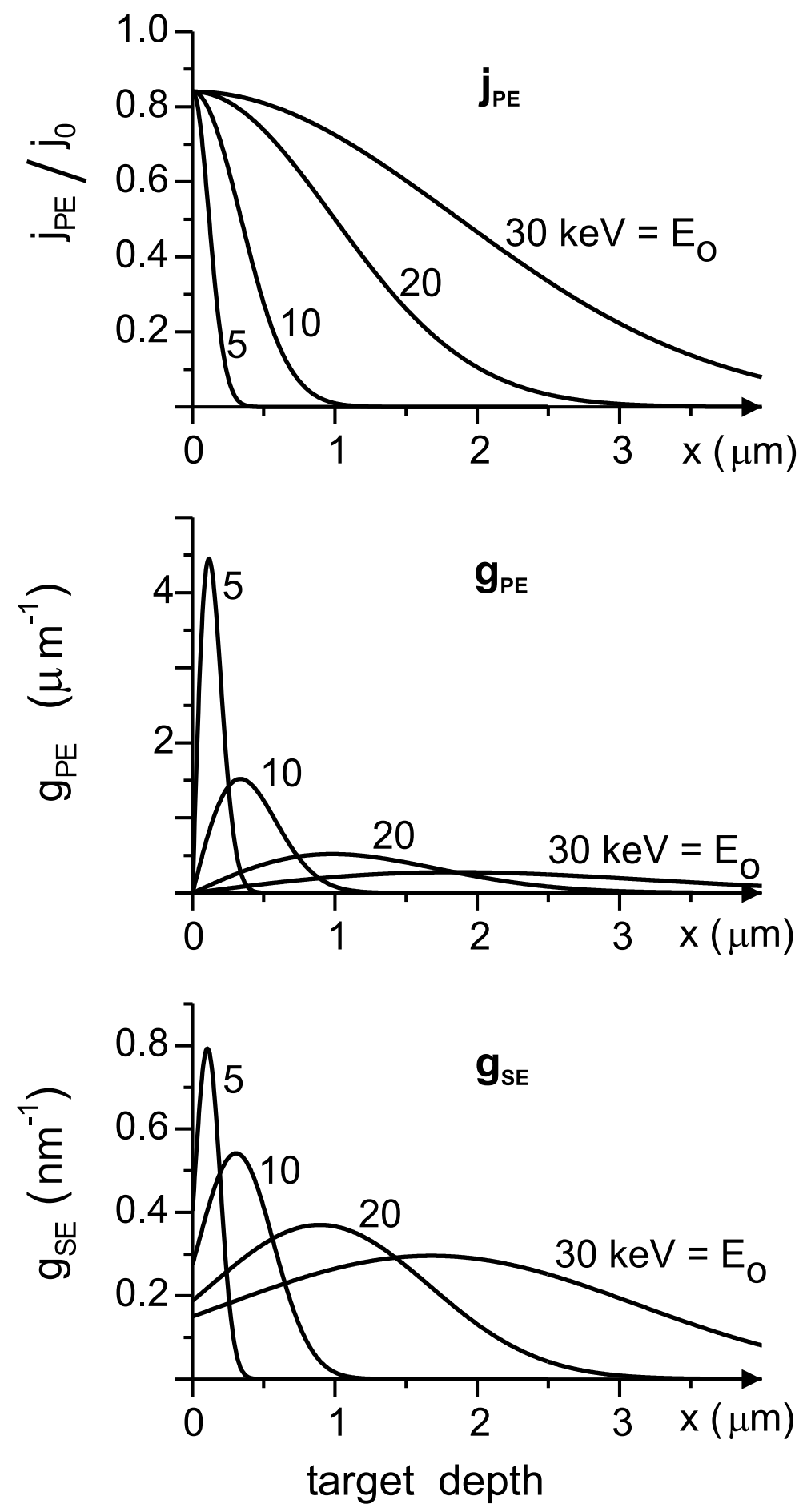

Fig. 3 Current $j_{\mathrm{PE}}(x)$ of primary electrons with initial energy $E_{0}$ into $\mathrm{Al}_{2} \mathrm{O}_{3}$, their deposition (absorption) function $g_{\mathrm{PE}}(x)$, and their secondary electron generation $g_{\mathrm{SE}}(x)$, according to Eqs. (4) - (9), respectively. 


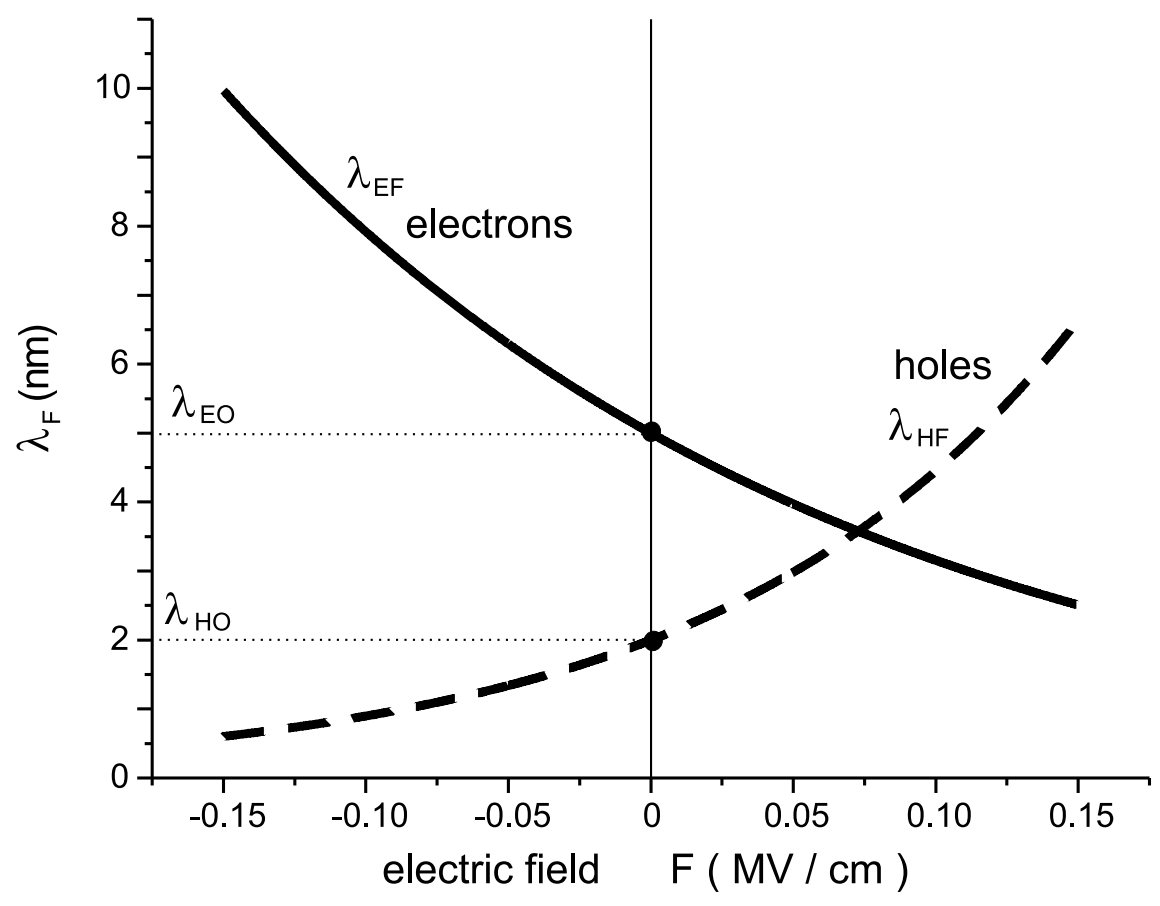

Fig. 4 Mean attenuation lenghts of electrons $\lambda_{\mathrm{EF}}$ and holes $\lambda_{\mathrm{HF}}$ in $\mathrm{Al}_{2} \mathrm{O}_{3}$ as a function of the electric field $F$, according to Eq. (11). 
$\mathrm{PE}$

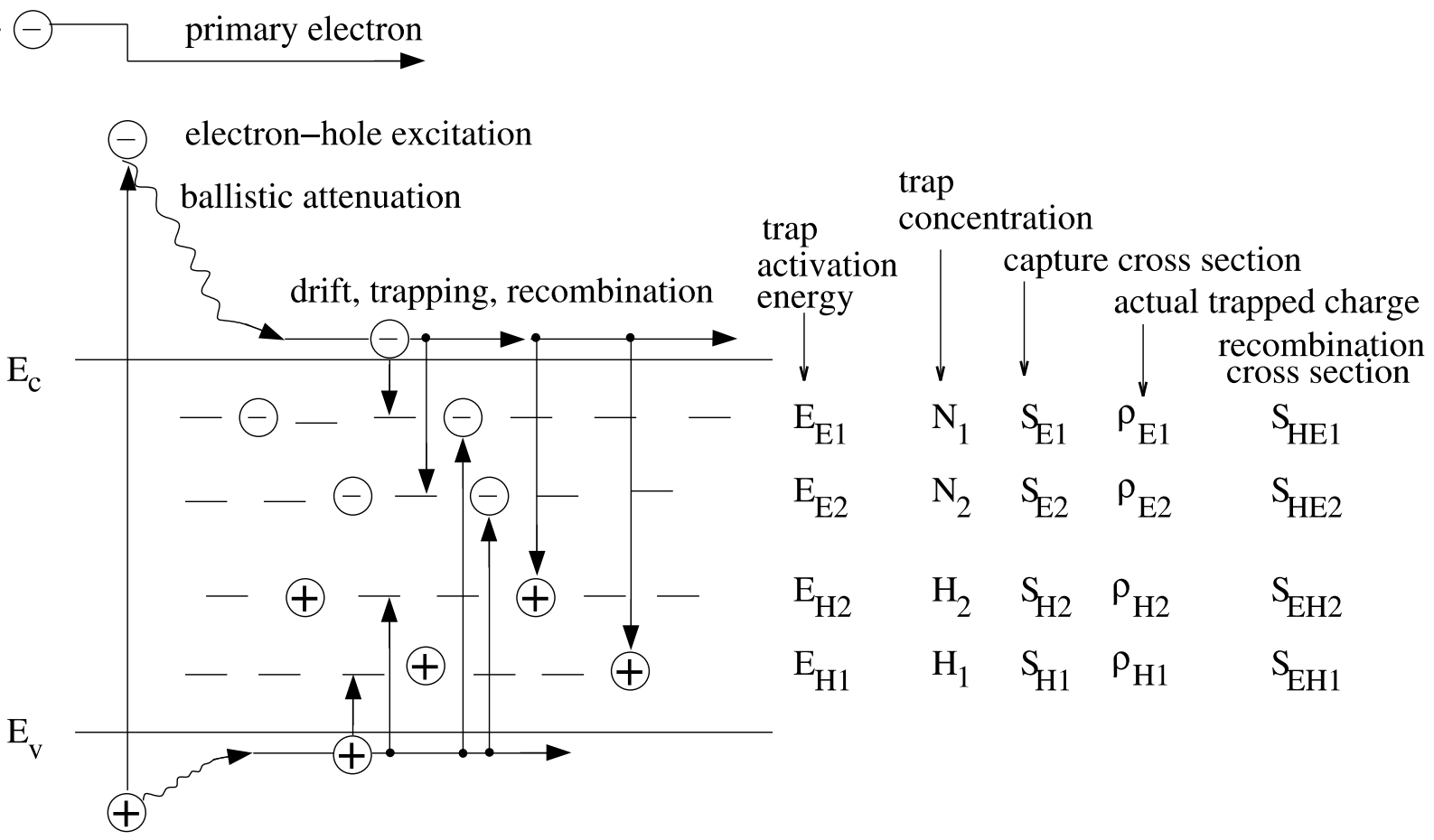

Fig. 5 Scheme of the flight-drift model including the ballistic flight and attenuation followed by drift, trapping, and recombination of electrons and holes. 


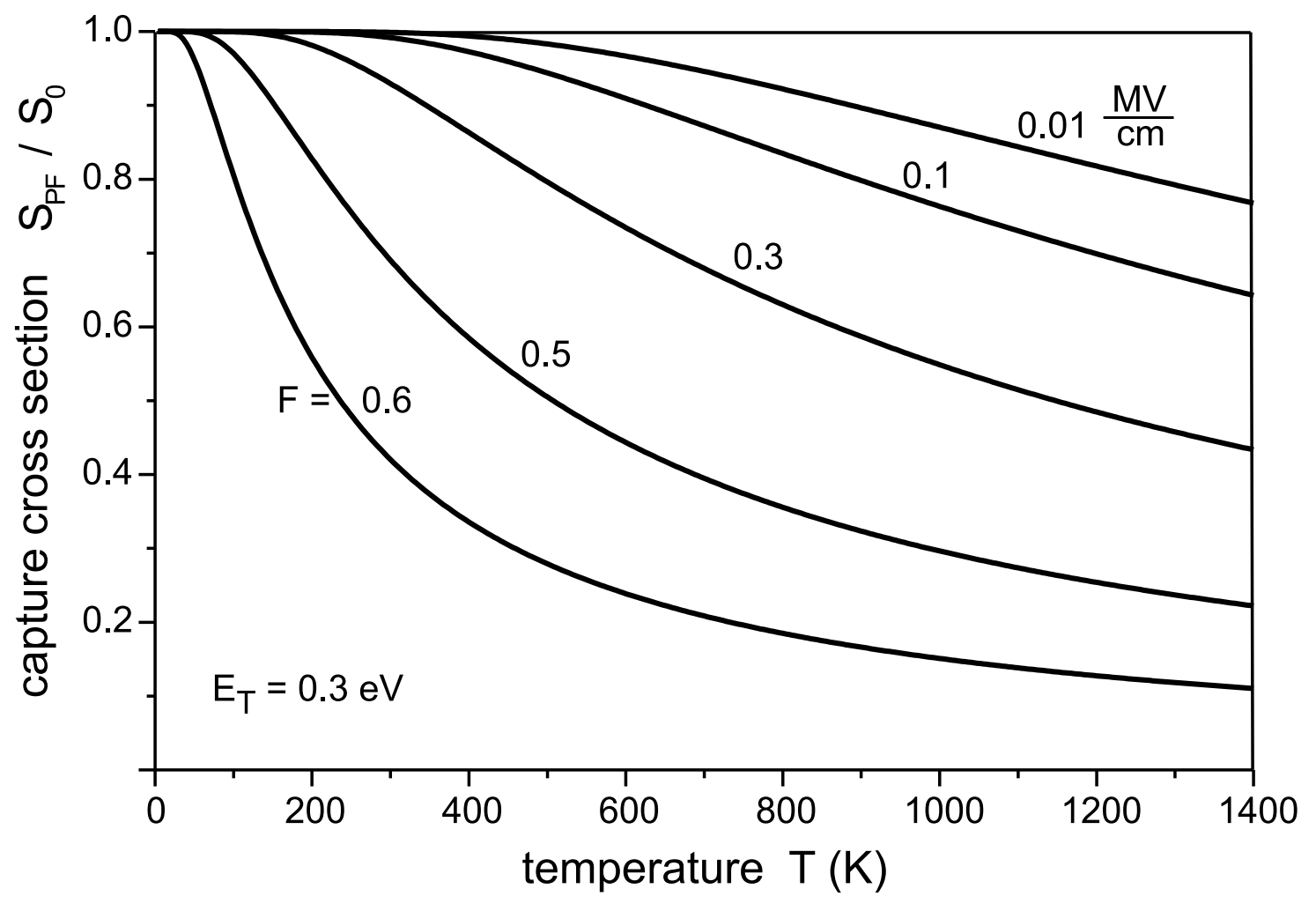

Fig. 6 Poole-Frenkel-modified capture cross sections $S_{\mathrm{PF}}$ according to Eq. (25) of a shallow trap with thermal activation energy $E_{T}=0.3 \mathrm{eV}$ in dependence on temperature $T$ and electric field $F$. 


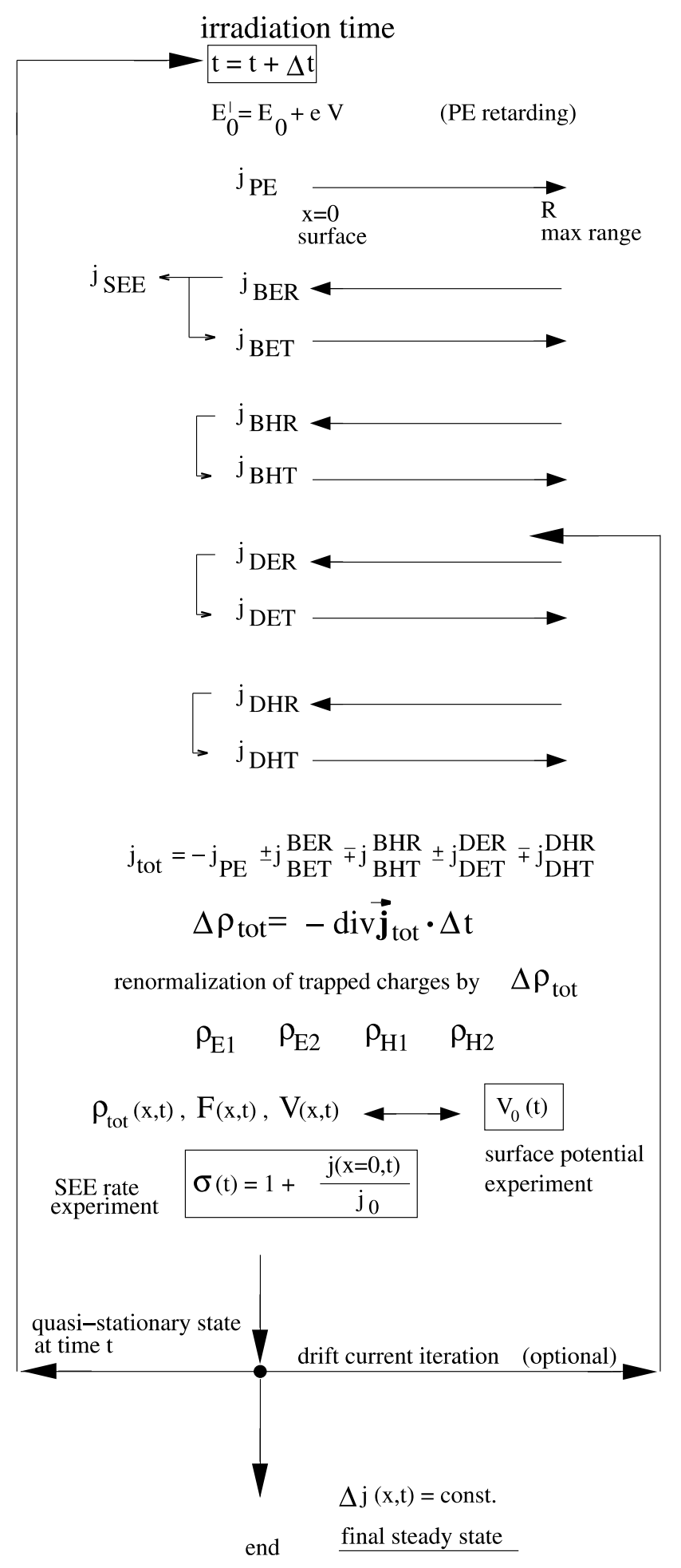

Fig. 7 Computation scheme of the selfconsistent charging in insulating samples. 
A : Open Layer ; $E_{0}=20 \mathrm{keV}$
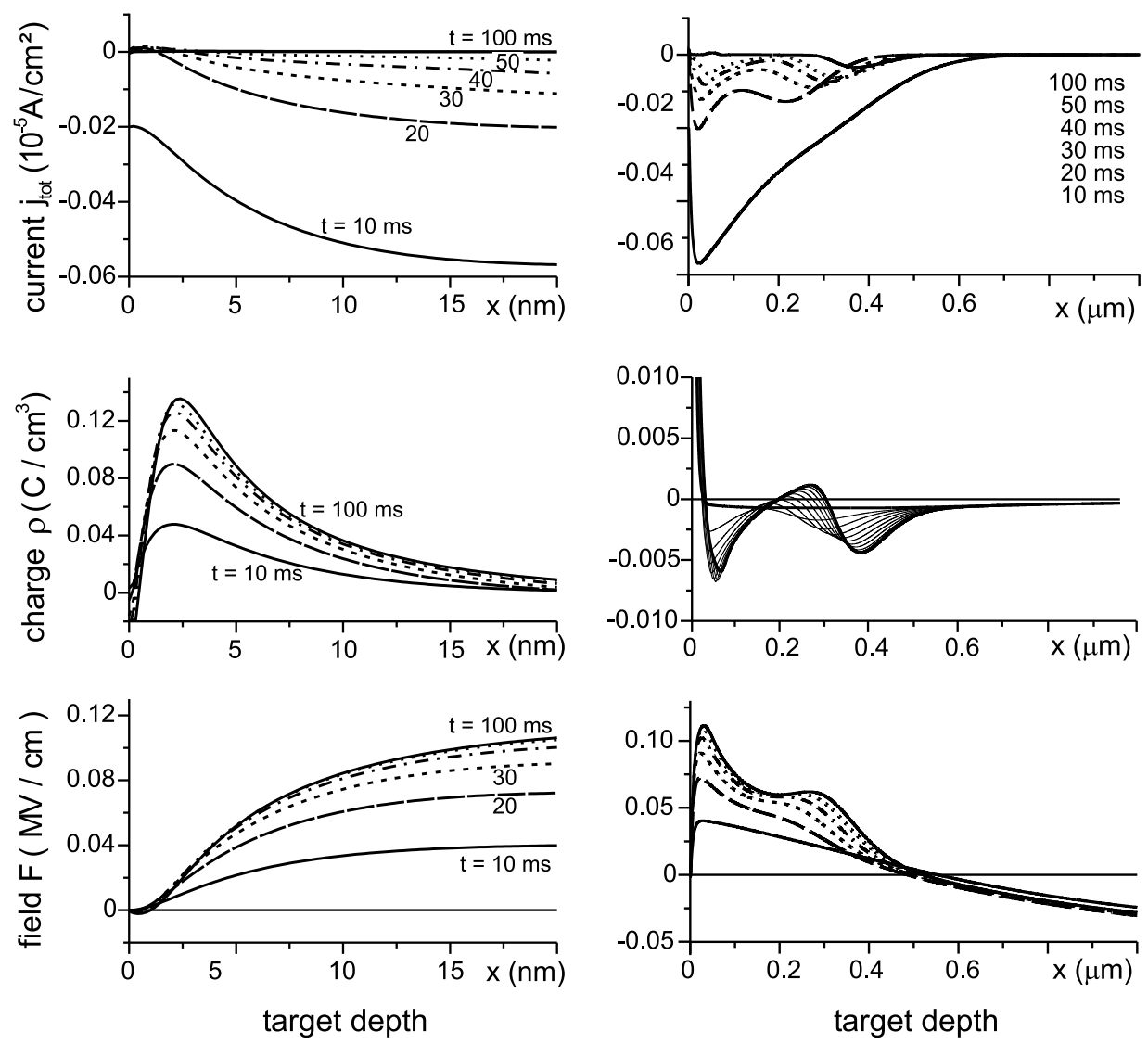

Fig. 8 Total current $j_{\text {tot }}(x)$, charge $\rho(x)$, and field $F(x)$ depth distributions as a function of irradiation time $t$ for an initial beam energy of $E_{0}=20 \mathrm{keV}$ and current density $j_{0}=10^{-5} \mathrm{~A} / \mathrm{cm}^{2}$; left: benath the surface zoomed in $\mathrm{nm}$ scale; right: in the bulk alumina in $\mu \mathrm{m}$ scale. Mention that all the bulk distributions are shrinked towards the surface because of a strong negative charging and respective retarding of the electron beam to $E_{0}^{\prime}=E_{0}-e V_{0}=7 \mathrm{keV}$ with much less electron ranges already after 10 ms irradiation time. 

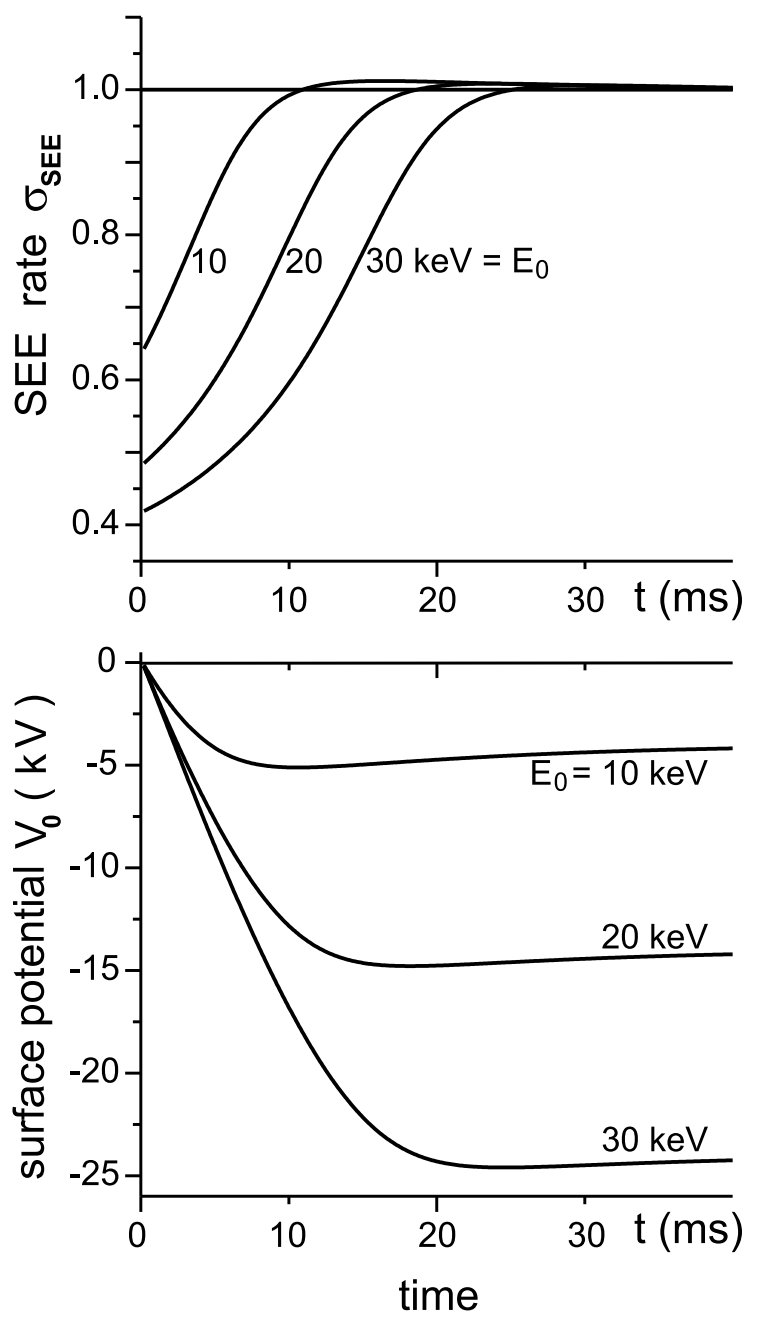

Fig. 9 Secondary electron emission rate $\sigma$ and surface potenial $V_{0}$ of bulk alumina targets as function of irradiation time $t$ for different electron beam energies $E_{0}: j_{0}=10^{-5} \mathrm{~A} / \mathrm{cm}^{2}$. 


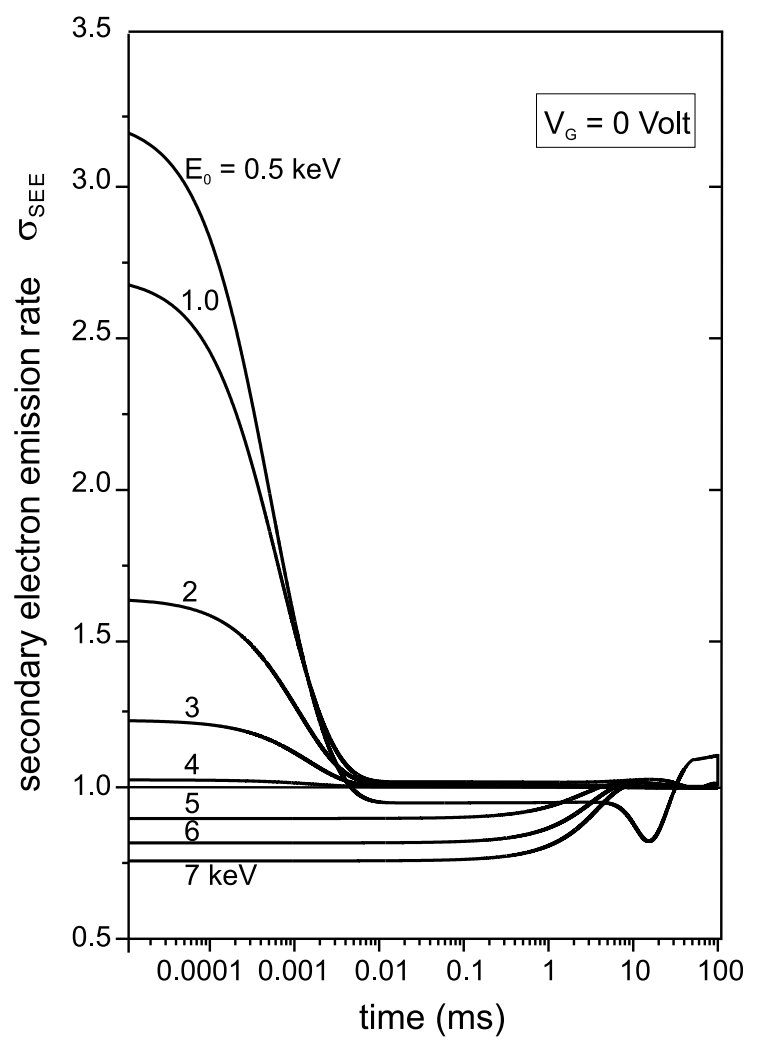

Fig. 10 Secondary electron emission rate $\sigma_{\mathrm{SEE}}=\eta+\delta$ in dependence on irradiation time for different $\mathrm{PE}$ energies $E_{0}=(0.5-7) \mathrm{keV}$ in the transition region $\sigma \gtreqless 1$; $j_{0}=10^{-5} \mathrm{~A} / \mathrm{cm}^{2}$. 


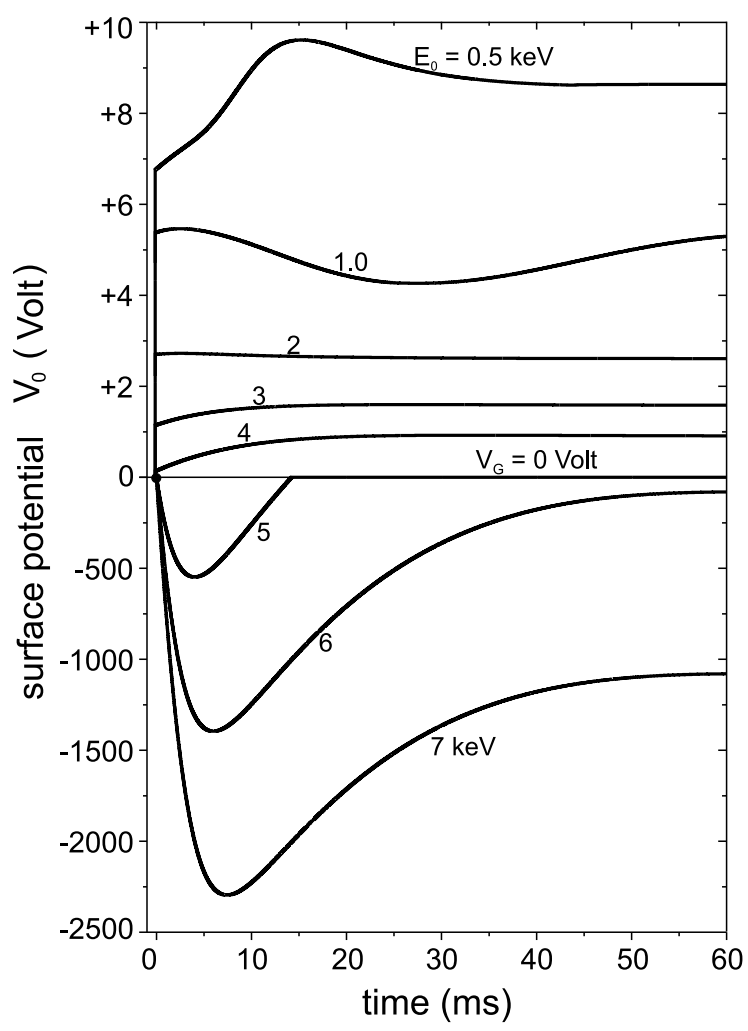

Fig. 11 Surface potential $V_{0}$ in dependence on irradiation time for different PE energies $E_{0}=(0.5-7) \mathrm{keV}$ in the transition region $\sigma \stackrel{\gtrless}{\gtrless} ; j_{0}=10^{-5} \mathrm{~A} / \mathrm{cm}^{2}$. 


\section{Surface Reflection - Absorption}

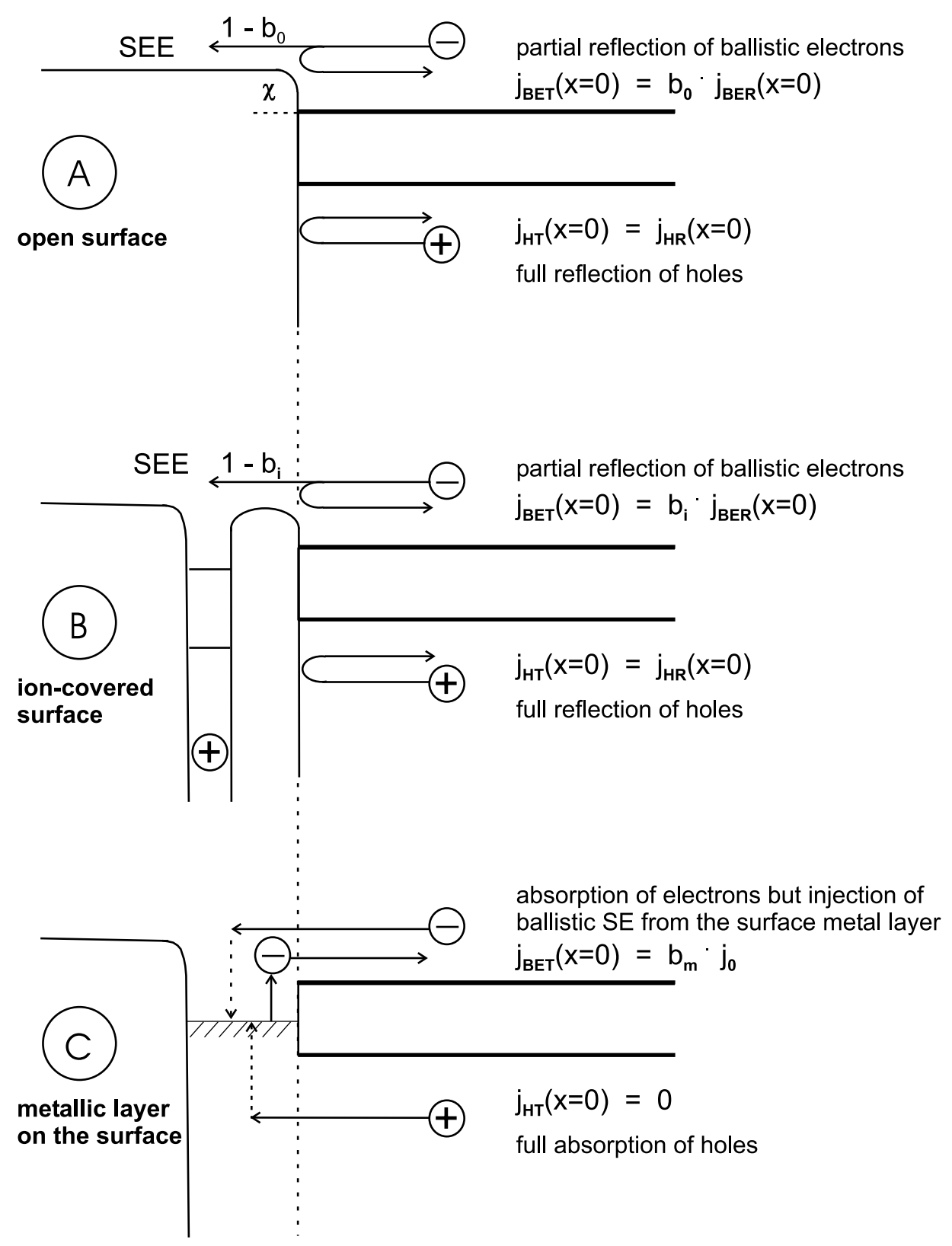

Fig. 12 Surface reflection and/or absorption of electrons and holes at: A: open surface;

B: positive-ion-covered surface, and C: metallized and grounded surface. 
(B): lon-covered surface
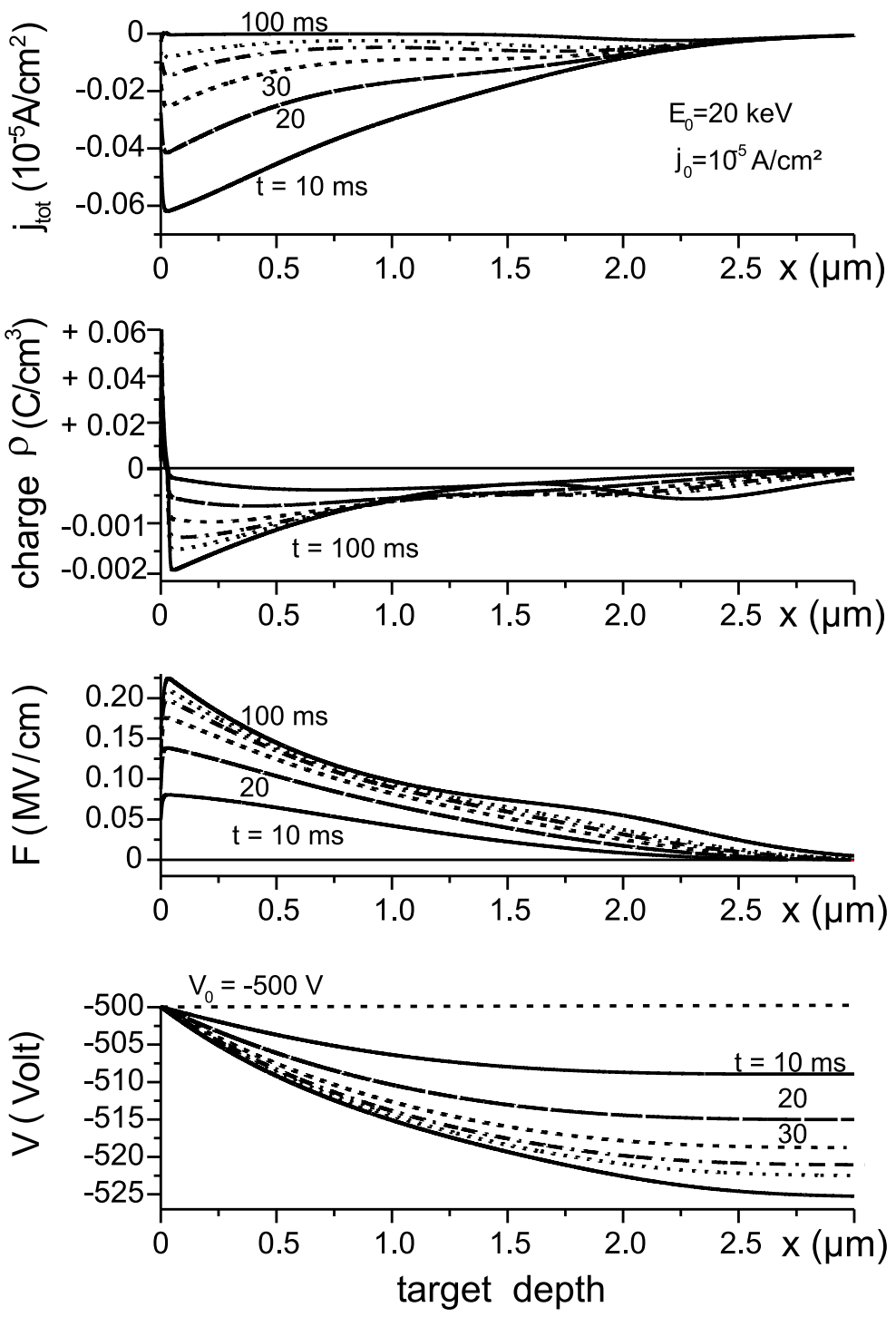

Fig. 13 Current $j_{\text {tot }}(x)$, charge $\rho(x)$, electric field $F(x)$, and potential $V(x)$ depth distributions beneath the positive-ion-covered surface $\left(V_{0}=-500\right.$ Volt $)$ of alumina in dependence on irradiation time $t$; electron beam energy $E_{0}=20 \mathrm{keV}$ and current density $j_{0}=10^{-5} \mathrm{~A} / \mathrm{cm}^{2}$. 
(C) : Metal-covered surface
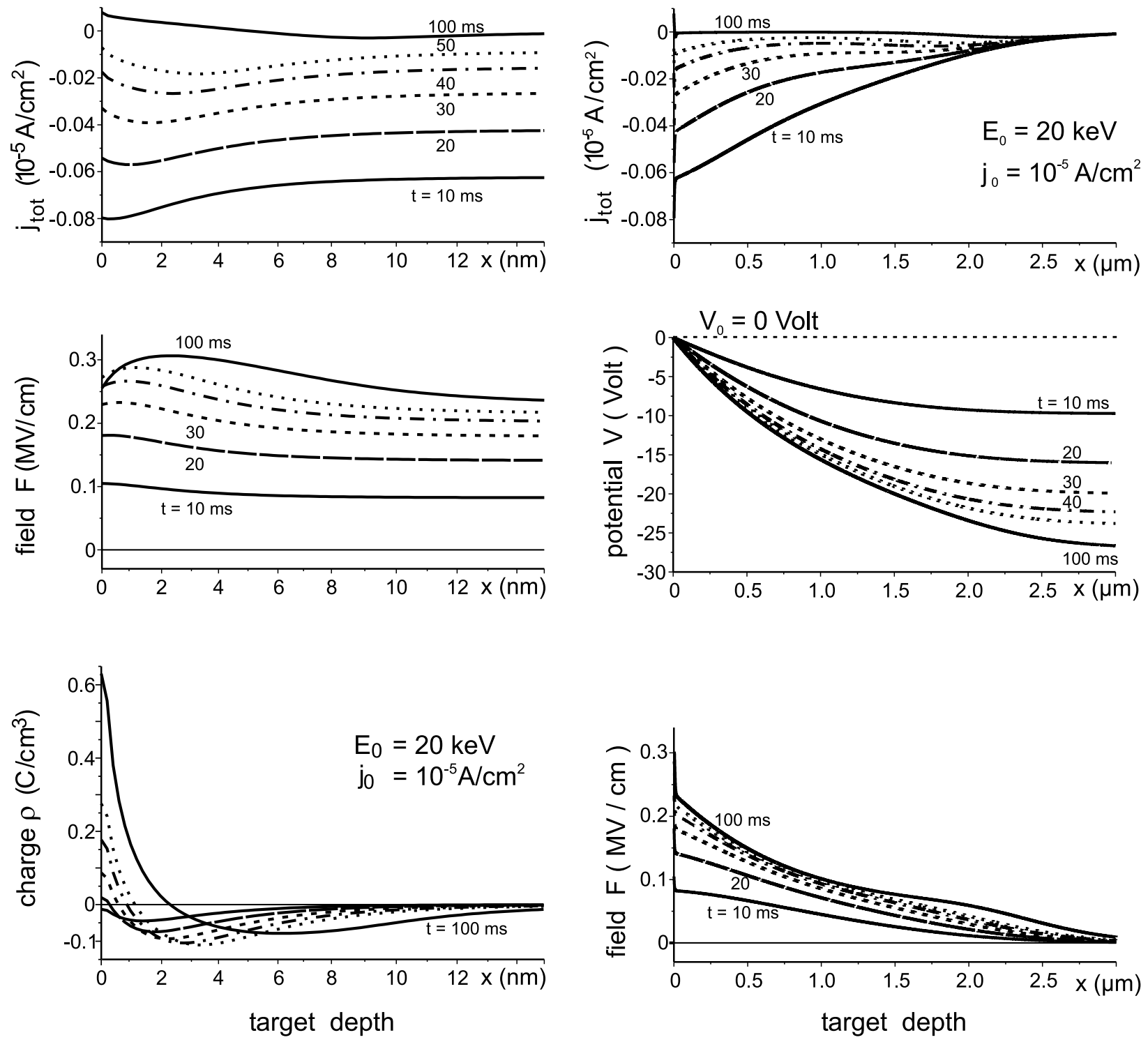

Fig. 14 Current $j_{\text {tot }}(x)$, charge $\rho(x)$, electric field $F(x)$, and potential $V(x)$ depth distributions beneath the positive metal-covered and grounded surface $\left(V_{0}=0\right)$ in dependence on irradiation time $t$, zoomed in nm scale (left) and presented in $\mu \mathrm{m}$ scale (right); electron beam energy $E_{0}=20 \mathrm{keV}$ and current density $j_{0}=10^{-5} \mathrm{~A} / \mathrm{cm}^{2}$. 